\title{
Adsorption of dye molecules on metallic nanoparticles: unraveling the origins of the modified spectral absorption
}

\author{
by \\ Xiaohan Chen
}

Supervisor

Eric Le Ru

\begin{abstract}
A thesis
submitted to the Victoria University of Wellington in fulfilment of the requirements for the degree of Master of Science in Chemical and Physical Sciences.
\end{abstract}

Victoria University of Wellington 2019 



\begin{abstract}
The enhanced optical response of molecules in the vicinity of metallic nanoparticle is the basis for many surface enhanced spectroscopies and of interest to the field of plasmonics. However, the mechanisms behind the enhancement are still a matter of debate because of the interplay between electromagnetic and chemical effects, which complicates the interpretation of spectral changes. Our ability to measure the surface absorption of dyes from very low coverage to high coverage allows us to identify the contribution of each effect (dye-dye interaction and dye-particle interaction) to the spectral modifications. In the course of this investigation, we also measured the adsorption isotherms of dyes in the presence of halide ions, which provides a detailed insight into the adsorption process on silver colloids.
\end{abstract}




\section{Contents}

1 Introduction 1

1.1 Background ..................... 1

1.2 Overview of the thesis $\ldots \ldots \ldots \ldots$

2 Integrating cavity-Theory and Experimental Setup $\quad 7$

2.1 The theory of integrating cavity .......... 8

2.1.1 Reflection from Lambertian Surface . . . . . . . . . 8

2.1.2 Sphere multiplier $-\mathrm{M}$ factor . . . . . . . . . . 9

2.2 Integrating sphere as absorption spectrometer . . . . . . . 12

2.2.1 Experimental Setup and Standard Absorbance Measurement Steps . . . . . . . . . . . . . . . 12

2.2.2 Absorbance correction . . . . . . . . . . . . 14 
2.2.3 Absorbance-concentration calibration . . . . . . 16

3 Adsorption properties of molecules on NPs 21

3.1 Experimental method . . . . . . . . . . . . . . 22

3.1.1 Sample preparation . . . . . . . . . . . 22

3.1.2 Absorption measurement . . . . . . . . . . 23

3.2 Results and discussions $\ldots \ldots \ldots \ldots$

3.2.1 Unneutral molecules wall adsorption . . . . . . . . 24

3.2.2 Adsorption of R6G to Silver Nanoparticles . . . . . . 26

3.2.3 Adsorption isotherms in halide solutions . . . . . . 29

4 Modified absorbance of R6G on silver 33

4.1 Experimental method . . . . . . . . . . . . . 34

4.1.1 Surface absorbance measurement in the integrating cavity . . . . . . . . . . . . . 34

4.2 Results and discussions . . . . . . . . . . . . . 34

4.2.1 Low-coverage surface absorption . . . . . . . . . 34

4.2.2 Concentration-dependent surface absorption . . . . . 37

4.2.3 Could it be chemical etching? . . . . . . . . . . 39 
CONTENTS

5 Conclusion 


\section{Chapter 1}

\section{Introduction}

\subsection{Background}

Metal nanoparticles - including gold, silver, and platinum - show very interesting optical properties and are highly efficient at absorbing and scattering light [1]. By changing nanoparticle size and shape the optical response can be tuned from the ultraviolet through the visible to the near-infrared regions of the electromagnetic spectrum. The reason for the unique spectral response of silver and gold nanoparticles is that specific wavelengths of light can drive the conduction electrons in the metal to collectively oscillate, a phenomenon known as a surface plasmon resonance (SPR) [1]. When these resonances are excited, absorption and scattering intensities can be largely enhanced compared to identically sized particles that are not plasmonic. Figure 1.1 shows the optical response of a $50 \mathrm{~nm}$ diameter silver nanosphere. One can see that its absorption and scattering has a maximum at $430 \mathrm{~nm}$, where the surface plasmon resonance occurs. 


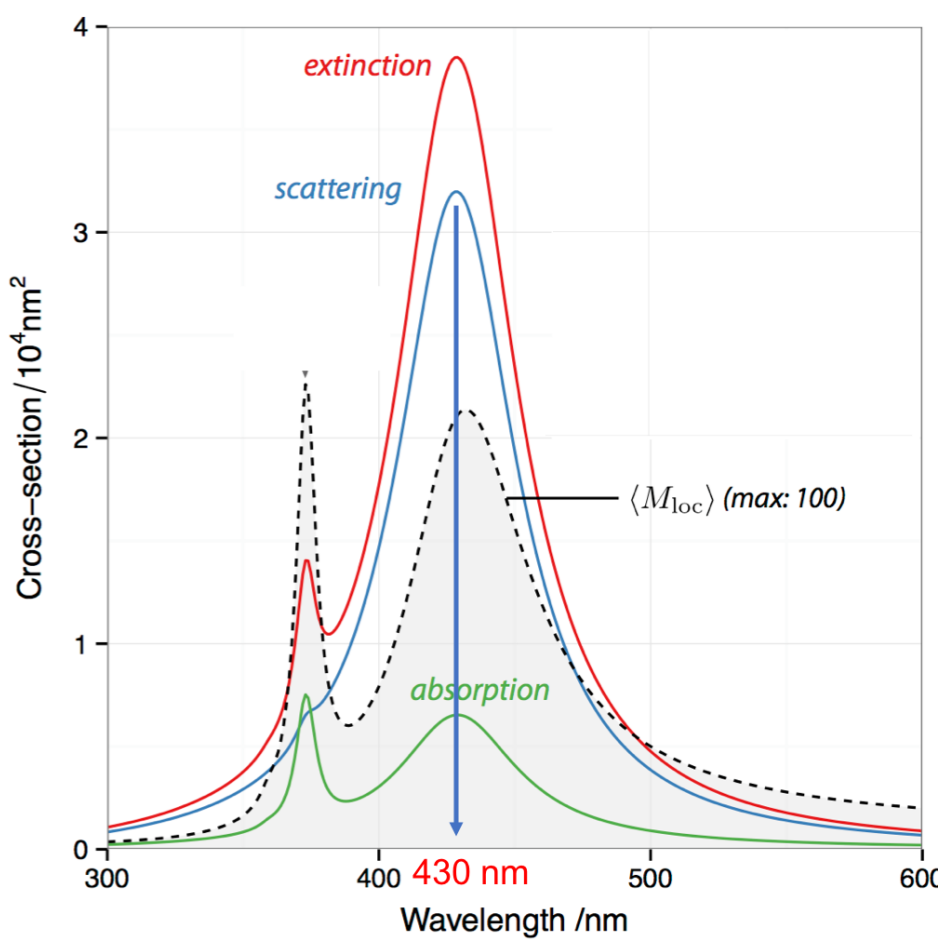

Figure 1.1: The absorption and scattering spectra of $50 \mathrm{~nm}$ diameter silver nanosphere, peaking at $430 \mathrm{~nm}$.

The interaction between the plasmon resonance of metallic nanoparticles/films and the electronic resonance of dyes adsorbed on their surface has been exploited and applied in many contexts: for example, the detection of single molecule [2,3], the enhancement of photochemical reactions on surfaces [4-6], improved photovoltaics [7, 8], and surface enhanced spectroscopies [1,9]. Many of these applications are underpinned by the fact that the optical absorption of dyes are largely enhanced in the vicinity of the metallic surface, where light/matter hybrid systems can be created. In these studies, the concentration of surface dyes are high, typically forming multilayers or J-aggregates to maximize plasmon/dye interaction. As a result, both the dye-plasmon and dye-dye interactions 
effects become important and the intrinsic adsorption induced absorption spectral change is hard to elucidate.

Previous work [10] by our group has shown the necessity of accessing the low dye concentration range $(10 \mathrm{nM})$ to minimize the dye-dye interaction. This is challenging with existing technologies since the absorbance is very low and obscured by the large response of nanoparticle. To address this, a promising method based on measuring the surface differential absorbance with an integrating cavity was developed.

An integrating cavity is an optical component whose main function is to uniformly distribute light through diffusive reflections. It has been widely applied as a standard instrument in photometric or radiometric measurements [11], whereby the light produced by a source and the total power can be obtained in a single measurement. A rare use of the integrating sphere is performed as UV-Vis spectrometer. Such a setup has been applied in the past $[12,13]$ to measure the absorption of seawater and microalgae [14-17] where scattering by suspended particles and the low concentrations of absorbing species can limit the use of a standard UV-Vis spectrometer. During previous work by our group, we developed a centrally mounted sample cuvette integrating sphere for the absorbance measurement of dyes molecules on colloidal nanoparticles [10]. Such a device provides advantages in measuring very diluted sample through an enlarged output by multiple reflections of light inside the sphere, thus allowing us to access sub-monolayer range of dye coverage on the particles. Modifications of the intrinsic dye polarizability upon adsorption on metallic nanoparticles have been evidenced [10].

Motivated by this, we in this study continue exploring the origins of the modified absorption of dyes in metallic colloid solutions. Specifically, we focus on one problem that has attracted much interest and generated 
debates over the years [18-20]: the effect of halide anions on the absorption of Rhodamine 6G (R6G) on silver nanoparticle (AgNP). Such a system has been widely studied in the context of Surface Enhanced Raman Spectroscopies (SERS) $[18,21]$, where an enhancement in the intensity of R6G is always observed in the presence of halide anions. However, the exact mechanism behind the enhancement is still a mater of debate, because of the difficulties in disentangling the electromagnetic effect and the chemical effect from each other.

In this study, I demonstrate explicitly the influence of the halide anions on the adsorption of R6G on AgNP by revisiting adsorption isotherms, which provides vital information about the chemical/physical interaction of dyes and the nanoparticles (NP). I also show that the concentrationdependent spectra provide insight into the dye-dye coupling on the sphere. These have been so far almost completely overlooked in discussions of plasmon-dye coupling experiments but our results indicate that they are important even at relatively low concentrations. These spectra additionally reflect the change in the dye orientation, which we show is impacted by halide anions.

Our experimental results are in good agreement with the predictions of a coupled-dipole model recently developed by our group [22]. We anticipate that our experimental findings will have important implications for the understanding of the interaction between NP and adsorbed molecules, and provide a deeper insight into the respective contributions of electromagnetic effects or those of a more chemical nature. 


\subsection{Overview of the thesis}

The work described in this thesis is experimental and it is aimed at unravelling the origins of the spectral changes in the absorption of dyes on metallic nanoparticles. To attempt this task we focus on one system which consists of colloidal silver and R6G dyes in the presence of various halide anions because it allows the elucidation of both the electromagnetic and chemical effects on the dye-dye and dye-NP interactions resulting in modifications in the absorption spectra.

The thesis progresses in the following way. In chapter 2, the working principle of the centrally mounted sample cuvette integrating sphere is discussed and the method for extracting the surface absorbance of dyes is introduced. In chapter 3, I show how the type of vessels for centrifugation affects the adsorption quantification of R6G molecules on silver sphere. I also demonstrate how the halide anions $\left(\mathrm{Cl}^{-}, \mathrm{Br}^{-}\right.$and $\left.\mathrm{I}^{-}\right)$influence the adsorption efficiency of dyes with adsorption isotherms. In chapter 4, by carrying out concentration-dependent differential absorption spectra, I elucidate the adsorption effects, including the dye-dye interaction and the dye orientation effects, on the spectral modifications. Finally, in the conclusion I summarize the achievements of the work described in this thesis, and suggest some possible future directions. Brief introductions into each area will be given at the beginning of each chapter. 


\section{Chapter 2}

\section{Integrating cavity-Theory and Experimental Setup}

In this chapter, we present the working principles of the integrating cavity. The main factors governing the working of this instrument are discussed along with its application in performing as a UV-Vis spectrometer for absorbance measurement. In particular, the necessity of the correction/calibration for the measured absorbance in the integrating sphere is addressed, and the specific correction processes are demonstrated with reference dye samples. 


\subsection{The theory of integrating cavity}

\subsubsection{Reflection from Lambertian Surface}

One principal function of an integrating cavity is to uniformly distribute light through diffusive reflections from its inner surface. In this process, the directional characteristic of the input light source is removed, leading to evenly distributed photons in the cavity after numerous reflections. The integrating cavity is a powerful instrument for optical measurements where the total power (flux) of a light source can be measured free from the effects of beam shape, incident direction, polarization, or the measurement device. The earlier applications of integrating cavity are mainly in photometry and radiometry [11,23], where the total radiation flux from a source is measured over all possible solid angle of emission.

By placing a lamp inside a diffusely reflecting integrating sphere, the detector placed at a port on the sphere can collect the total emitted flux because the brightness of the surface to an observer is the same regardless of the observer's angle of view [24]. The surface's luminance is isotropic through ideally diffusive reflections, which is the property that defines an ideal "matte" or diffusely reflecting surface, called a Lambertian surface or Lambertian reflector.

Figure 2.1 demonstrates the properties of a light ray propagating from the Lambertian surface. In (a), the incident ray $\mathrm{I}_{0}$ undergoes reflection from the surface and scatters light in all directions. The luminous intensity obeys Lambert's cosine law where the intensity is proportional to the cosine of the angle between the surface normal and scattering direction. The maximum intensity reflected will be at $\theta=0^{\circ}$ and decreases to 0 at $\theta=$ $90^{\circ}$. 

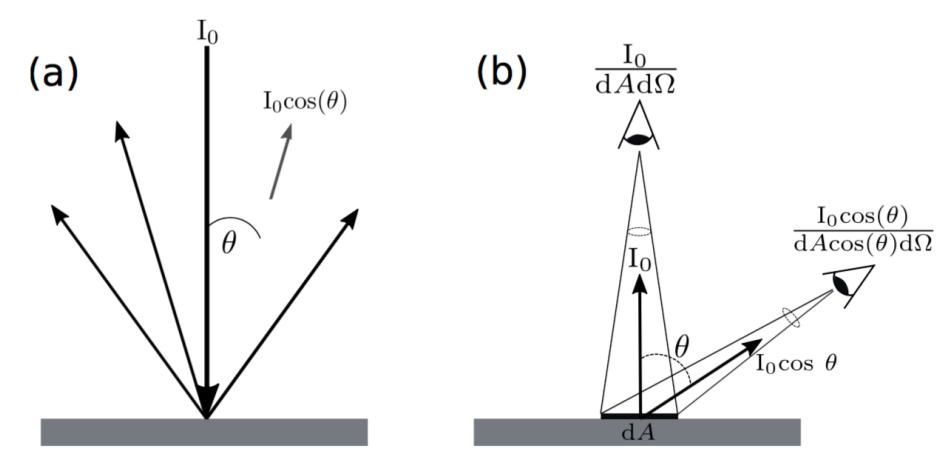

Figure 2.1: Illustrations of Lambertian reflection: (a)The intensity of a reflected ray from Lambertian surface follows Lamberts cosine law. (b) The projected area viewed by the observer also follows a cosine dependence, leading to the same radiance across the surface.

To further understand the function of Lambertian surface, we instead consider the radiance, the power emitted by unit area per solid angle $\left[\mathrm{W} / \mathrm{m}^{2} / \mathrm{sr}^{-1}\right]$ in the sphere. As shown in Fig.2.1 (b), the projected area into the observer also follows a cosine dependence, which compensates for the decrease in the intensity. As a result, the radiance on the surface is the same everywhere irrespective of the observation position.

\subsubsection{Sphere multiplier - $\mathrm{M}$ factor}

Figure 2.2 describes a single light ray undergoing multiple reflections in an integrating sphere with an entrance port and an exit/detection port. A major result of the multiple reflections is the increase in radiance, which can be referred to as the sphere multiplier M. Much of the theory of integrating cavity is well laid out in the Labsphere technical guide and has been discussed in details in [24]. The most important parameters characterizing the integrating sphere are listed below: 
- Sphere wall reflectivity: $\rho$.

- Port fraction $\mathrm{f}$, the ratio of total ports area to the sphere surface area: $\mathrm{f}=\mathrm{A}_{\text {ports }} / \mathrm{A}_{\text {sphere }}$.

- Sphere multiplier M: the gain in photon flux in comparison to the input power.

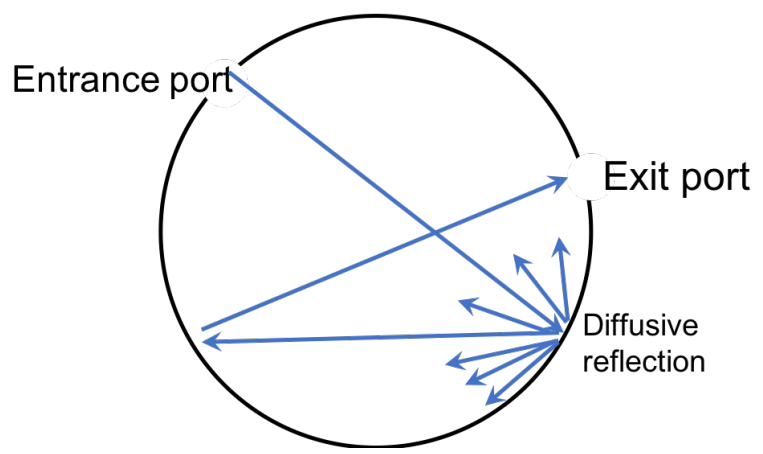

Figure 2.2: Depiction of the reflection path that a single ray undergoes in an integrating sphere. In simple terms, a light ray will undergo on average $M$ reflections before it exits the surface, which therefore increases the sphere radiance by a factor of $M$, called the sphere multiplier.

Simple reasoning behind the sphere multiplier $\mathrm{M}$ can be described as follows. When considering a photon undergoing multiple reflections inside the sphere, the probability of it surviving between each reflection/bounces equals $\rho(1-f)$. The first term corresponds to the probability for the photon to reflect off the sphere surface rather than being absorbed, and the second term is the probability of not exiting via a port. In terms of the perceived power of light, this indicates a decrease in the intensity by a factor of $\rho(1-\mathrm{f})$ after each reflection and gives: $\mathrm{I}_{n+1}=\rho(1-\mathrm{f}) \mathrm{I}_{n}$. As a result, the total intensity $\mathrm{I}\left[\mathrm{W} / \mathrm{m}^{2}\right]$ received at any points on the sphere surface is the sum:

$$
I=\sum_{n=1}^{\infty} I_{n}=I_{1} \sum_{n=1}^{\infty}\left[\rho(1-f)^{n}\right]=\frac{\rho}{1-\rho(1-f)} I_{0}
$$


The sphere multiplier is therefore defined as

$$
M=\frac{\rho}{1-\rho(1-f)}
$$

The multiplier $\mathrm{M}$ accounts for the increase/gain in photon flux in comparison to the input power for a sphere of a given radius, reflectivity and port fraction. It can also be understood as the average number of reflections for a given photon/ray. For an empty sphere, the $\mathrm{M}$ factor is decided by its geometric properties as well as the reflectivity of the sphere. As indicated by equation 2.1 , a large reflectivity $\rho$ could effectively promote the performance of sphere by an enhanced output, and therefore improve the detection limit. The commercially available spheres tend to be made of diffuse materials that are highly reflective in the wavelength range of interest. The most common material available is Spectralon, which exhibits an average reflectivity of $99 \%$ in the visible range [25].

In the following sections, I will show how the integrating sphere can be used as a UV-Vis spectrometer to measure the absorbance of samples. In such a case, the $\mathrm{M}$ factor is additionally affected by the absorbance/scattering effects of the sample, which can be exploited to determine the absorbance of the sample. A larger value of $\mathrm{M}$ for the empty sphere leads to a longer interaction path length of light with the sample and provides an advantage towards measuring low concentrations of absorbing species. The cavities we designed for these experiments had $\mathrm{M}$ factors ranging between 40 and 150. 


\subsection{Integrating sphere as absorption spectrome- ter}

\subsubsection{Experimental Setup and Standard Absorbance Mea- surement Steps}

Integrating sphere has been widely applied for measuring the diffuse reflectance of opaque (solid) samples. With integrating spheres, measurement is performed by placing the sample in front of the incident light, held in a vertical position against the side of the sphere, and concentrating the light reflected from the sample into the detector [24]. The collected light becomes the reflectance (relative reflectance) with respect to the reflectance of the reference standard white board, which is taken to be $100 \%[24]$. Such set-up is also useful for measuring the UV-Visible absorption spectrum of diffusely transmitting materials and the reflectance spectrum, including quantitative color measurements, of diffusely reflecting materials [26]. A less common application of integrating cavity is as UV-Vis spectrometer to measure the absorbance of samples. The fact that it works independently of the scattering effect provides an advantage for measuring turbid samples/suspensions. Moreover, the increased path length of the light interacting with the sample permits an enlarged output and thus improves the detection limit. In this work, a centrally mounted cuvette integrating sphere setup is applied for absorbance measurement, and its working schematic is shown in Fig.2.3. Similar set-ups have been used before in the context of sea-water measurements [15-17] but we have here optimized it for our absorbance measurements along the lines of Ref. [10].

This experimental setup consists of an integrating sphere in which a central mounted cylindrical glass vial inserted into the sphere through a 


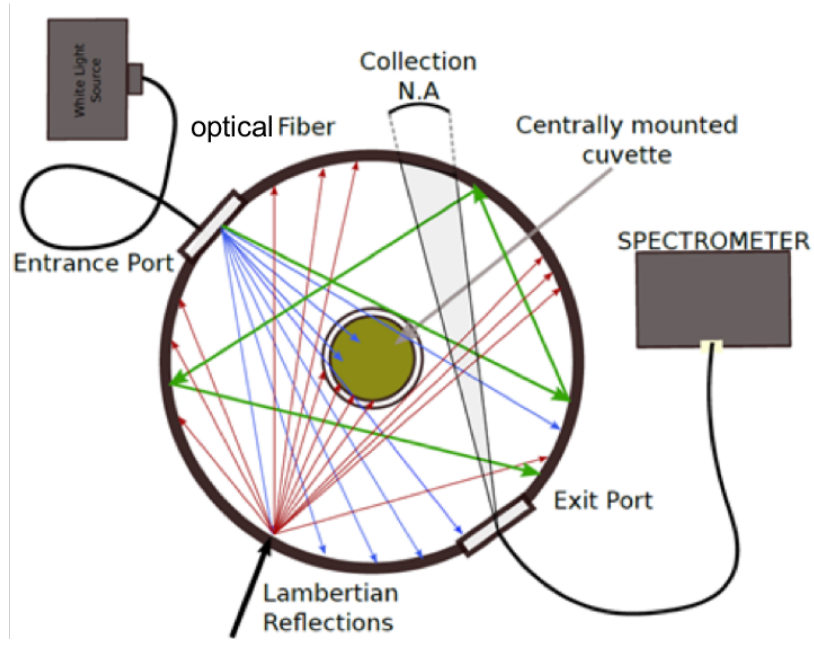

Figure 2.3: Schematic of the experimental setup. The possible incident/reflection paths of the light that undergoes in the sphere with entrance and exit ports.

port on top. The white light source is provided by a $100 \mathrm{~W}$ halogen lamp and delivered by an optical fiber through the entrance port. The transmitted light from the sample is collected via another optical fiber $(910 \mu \mathrm{m}$ core, $0.22 \mathrm{NA}$ ) that is inserted into the exit port, and coupled to an Ocean Optics USB-2000+ spectrometer.

A standard absorbance measurement consists of measuring the transmitted light of both the sample ( $\left.\mathrm{I}_{\text {sample }}\right)$ and the reference $\left(\mathrm{I}_{\text {reference }}\right)$. An example of the transmitted lamp spectra with a water solution and Rhodamine 6G (R6G) solution in water in the sphere is shown in Fig. 2.4(a). The measured absorbance spectrum of R6G is then obtained as in standard UV-Vis from:

$$
A_{\text {meas }}=-\log _{10} \frac{I_{\text {sample }}}{I_{\text {reference }}}
$$

where $\mathrm{I}_{\text {sample }}$ and $\mathrm{I}_{\text {reference }}$ are the intensities of transmitted light for sample and reference solutions. 
The exact experimental configurations used in our absorbance measurements (cavity geometry, port location and size, etc.) are not given in this thesis because of their commercial sensitivity (the start-up Marama Labs was spin-out from this research and aims to commercialize instruments carrying out such absorbance measurements).
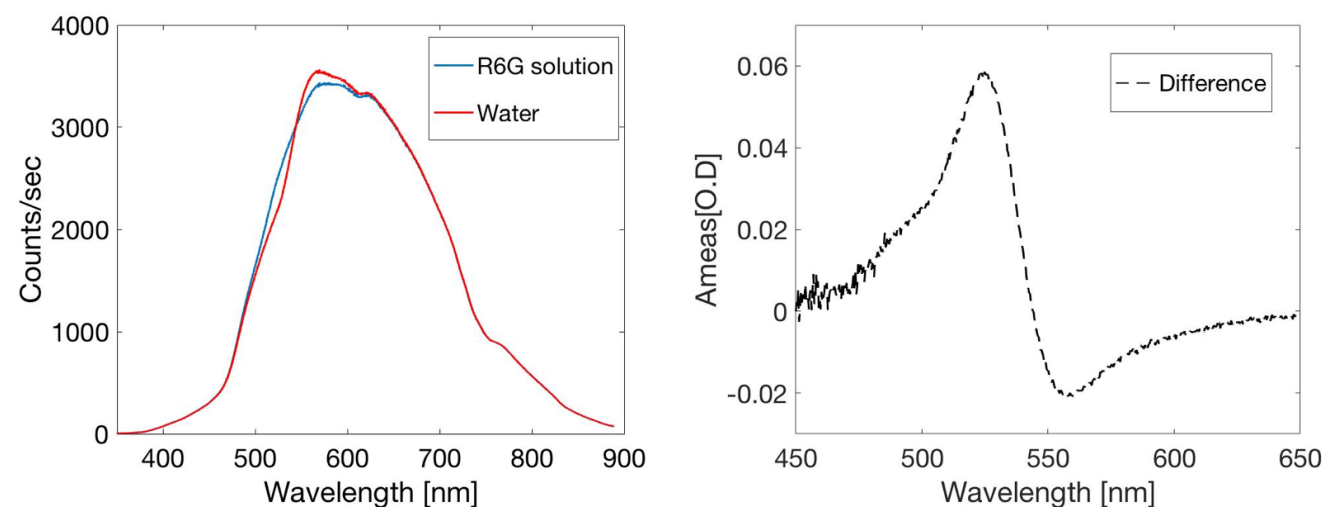

Figure 2.4: (a)Raw spectra of halogen lamp with water and 50 nM R6G solution in the sphere. The Halogen light $(100 \mathrm{~W})$ is coupled to a $1000 \mu \mathrm{m}$ core Fiber. (b) Differential absorption spectrum of the dye obtained from equation 2.3. The negative peak at $555 \mathrm{~nm}$ is due to fluorescence.

\subsubsection{Absorbance correction}

Comparing with the standard UV-Vis spectrometer, the absorbance measurement with the integrating sphere configuration is a lot more complicated because of the multiple reflections of light. For a standard UV-Vis setup, the incident light is shone directly through the sample of a given length and the transmitted light is directly collected by a detector. The absorbance of the sample then follows the Beer-Lambert Law:

$$
A=-\log _{10} \frac{P_{\text {transmit }}}{P_{\text {input }}}=c \varepsilon L
$$


where the $\mathrm{P}_{\text {transmit }}$ and $\mathrm{P}_{\text {input }}$ are the transmitted and input power of light; $c[\mathrm{M}]$ is the sample concentration; $\varepsilon\left[\mathrm{M}^{-1} \mathrm{~cm}^{-1}\right]$ is the molar extinction coefficient; $L[\mathrm{~cm}]$ is the path length through the sample, which in standard UV-Vis is well defined and typically $1 \mathrm{~cm}$. One can see that the obtained absorbance of the sample not only depends on its own optical property but is also influenced by the path length of the configuration. For the convenience of comparison, we propose to use the absolute absorbance $\left[\mathrm{cm}^{-1}\right]$, which can be converted from the raw absorbance A by normalizing with the path length.

In the sphere, a light ray would undergo numerous reflections and may therefore cross the sample several times from different random directions, therefore the total path length of the light interacting with the sample is largely increased. Theoretically, the path length inside the empty sphere can be calculated as the product of $\mathrm{M}$ factor (average number of reflections) and the average cord length for a sphere $\left(\mathrm{ML}_{\text {cord }}\right)$. The path length inside the sample is more complicated to predict, but will also be proportional to $\mathrm{M}$. However, the $\mathrm{M}$ factor is very difficult to determine in practice as it varies with both the wavelength (through reflectivity $\rho$ ) and the absorbance of the sample. A more practical way to normalize the measured absorbance and obtain the absolute absorbance is by direct comparison with the standard UV-Vis measurements for a reference non-fluorescing absorber of varying concentration. The ratios of absorbance given by the two methods should reveal how the path length changes inside the sphere and as a function of measured absorbance.

Series of mixed food dye samples were prepared by successive dilutions, and their absorbance was measured in the sphere (Fig. 2.5(a)) and a standard UV-Vis setup (Fig. 2.5(b)) in parallel. The food dye samples consist of four different dyes, with original concentrations of $500 \mathrm{mM}$. The initial mixed food dye sample is then prepared with a proportion of 17 
Tartrazine : 17 Sunset Yellow: 34 Indigo Carmine: 10 Eosin. Such a combination gives a 'flat' absorption spectrum, where the absorptivity across the visible region is similar. The other advantages of food dyes is that they are widely available, non-toxic, non-fluorescent, and negatively-charged. The latter reduces dilution errors due to adsorption to the walls.

The absorbance at several wavelengths $(\lambda=405,455,505,555,605$ and $655 \mathrm{~nm}$ ) was selected for calibration. The relation between the measured and the absolute absorbance is shown in Fig. 2.5(c): a linear relation is observed in the lower range of absorbance, while it starts to deviate and saturate as the absorbance increases. The slope at a certain point on the calibration curve equals the path length $[\mathrm{cm}]$ at that absorbance. We further plot the path length versus absolute absorbance, as shown in Fig. 2.5(d). It should be noted that the results of absorbance below 0.001 $\left[\mathrm{cm}^{-1}\right]$ are not reliable because of our limit of detection. Beyond that range $\left(\mathrm{A}_{\text {real }}>0.001\right)$, one can see that the measured increase in path length due to the integrating sphere was found to be approximately constant at $15 \mathrm{~cm}$ at $\mathrm{A}_{\text {real }}=0.01$, and decreases fast at higher absorbance. For experiments that require qualitative analysis (such as chapter 4 ), a fixed path length at the lower absorbance range can be applied to the conversion of the spectra obtained from the integrating sphere into a corresponding standard absorbance with little influence on the results. However, for samples with high absorbance, one needs to use the exact path length, or a direct equation to relate the measured and the 'real' values.

\subsubsection{Absorbance-concentration calibration}

The method of normalizing the absorbance with an approximate path length is straightforward and proved to be sufficient for the qualitative analysis 
of spectral changes/modifications or a rough estimation of concentration. However, for experiments that require an accurate quantification, such as the deduction of species concentration, or the enhancement factor for a particular optical process, this normalization approach is not appropriate and may lead to errors due to the nonlinear response of sphere.

Normally, the concentration of an absorbing solution can be easily obtained by measuring its absorbance with a standard UV-Vis setup. According to Beer Lambert law, the concentration is proportional to the absorbance for a given path length, normally $1 \mathrm{~cm}$. For measurements in an integrating sphere, the concentration of the sample cannot be estimated directly from the absorbance because of their nonlinear relationship. To address this carefully for our system of interest (Rhodamine 6G in water), we carried out systematic absorbance measurements on samples of known concentrations (see Fig. 2.6). The obtained experimental data (absorbance, concentration) is then fitted with a power function: $y=a x^{b}$, with $a=903.6$ and $b=0.9131$, from which one can calculate the concentration of an unknown sample.

The absorbance of R6G solutions with different concentrations was measured, and their absorption spectra are shown in Fig. 2.6. One may notice the negative region at the long wavelengths (from $550 \mathrm{~nm}$ to 650 $\mathrm{nm})$, which is due to the fluorescence by R6G molecule. The maximum absorbance in the spectra (at peak $526 \mathrm{~nm}$ ) were taken for generating data points $\left(\mathrm{A}_{R 6 G}\right.$, Concentration), as shown in Fig. 2.7. These experimental data are fitted well with an exponential function, whereby the concentration of R6G can be obtained knowing the corresponding absorbance. This method of obtaining the concentration is applied to the quantification of the adsorbed dyes on the particle in chapter 3 . 

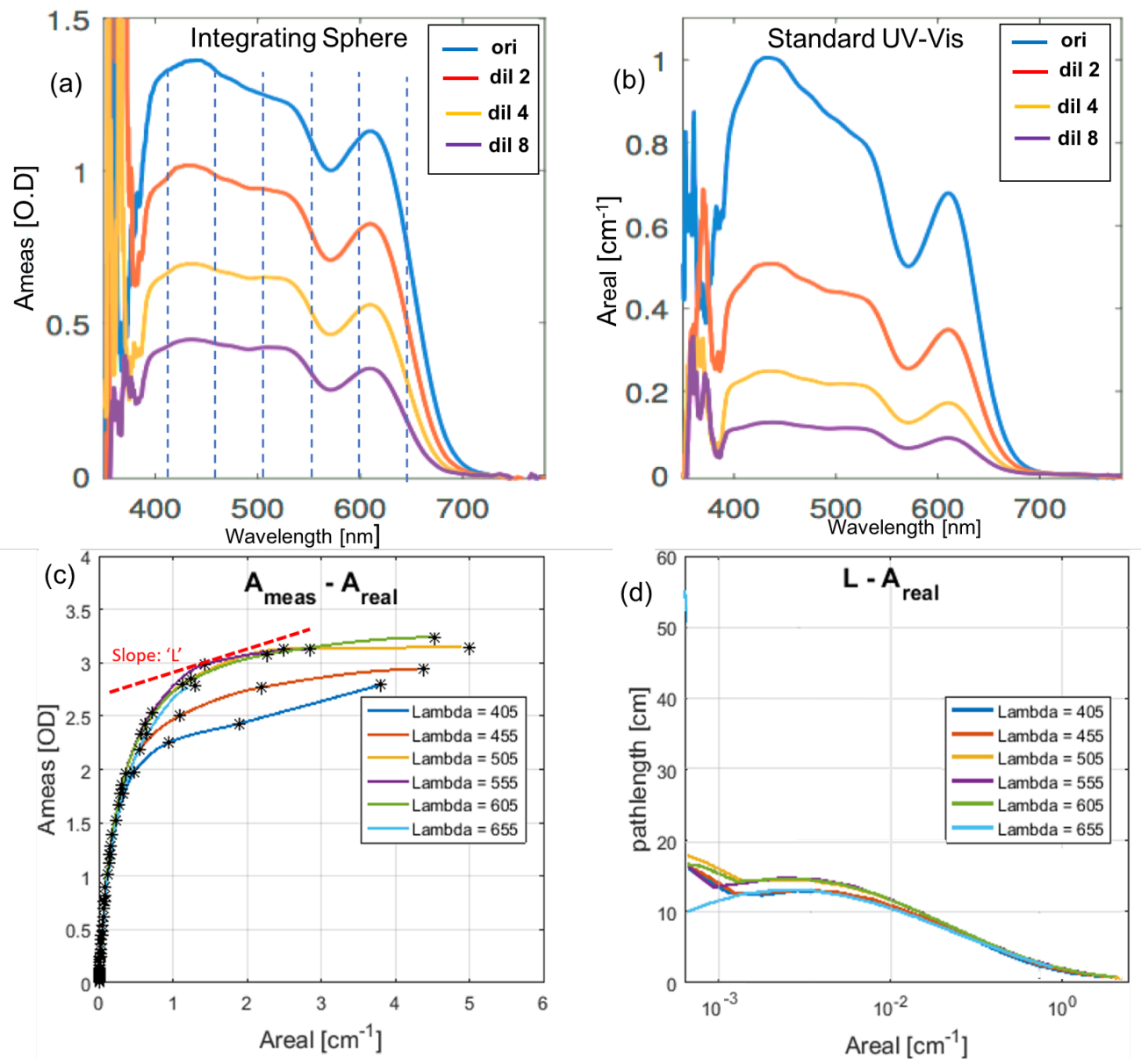

Figure 2.5: (a)(b) Absorbance spectrum of the mixed food dye samples measured in the integrating sphere (denoted $A_{\text {meas }}$ ) and a standard UV-Vis setup (denoted $\left.A_{\text {real }}\right)$ respectively. All the samples have the same constitutes except for the dilution factors: from 2 to 256. Only the first four spectra are shown here. (c) The experimental data (star points) $\left(A_{\text {real }}, A_{\text {meas }}\right)$ are connected smoothly. The relations between the measured and the standard (absolute) absorbance at different wavelengths are shown. (d) The effective path length in the integrating sphere, $L$, can be inferred from $L=A_{\text {meas }} / A_{\text {real }}$. Note that $L$ depends on the absorbance and the wavelength as shown in (d). Note that the apparent increase in L at low Areal is not real but due to the high uncertainty of the measurement for low absorbances (for example because of small baseline shifts). 


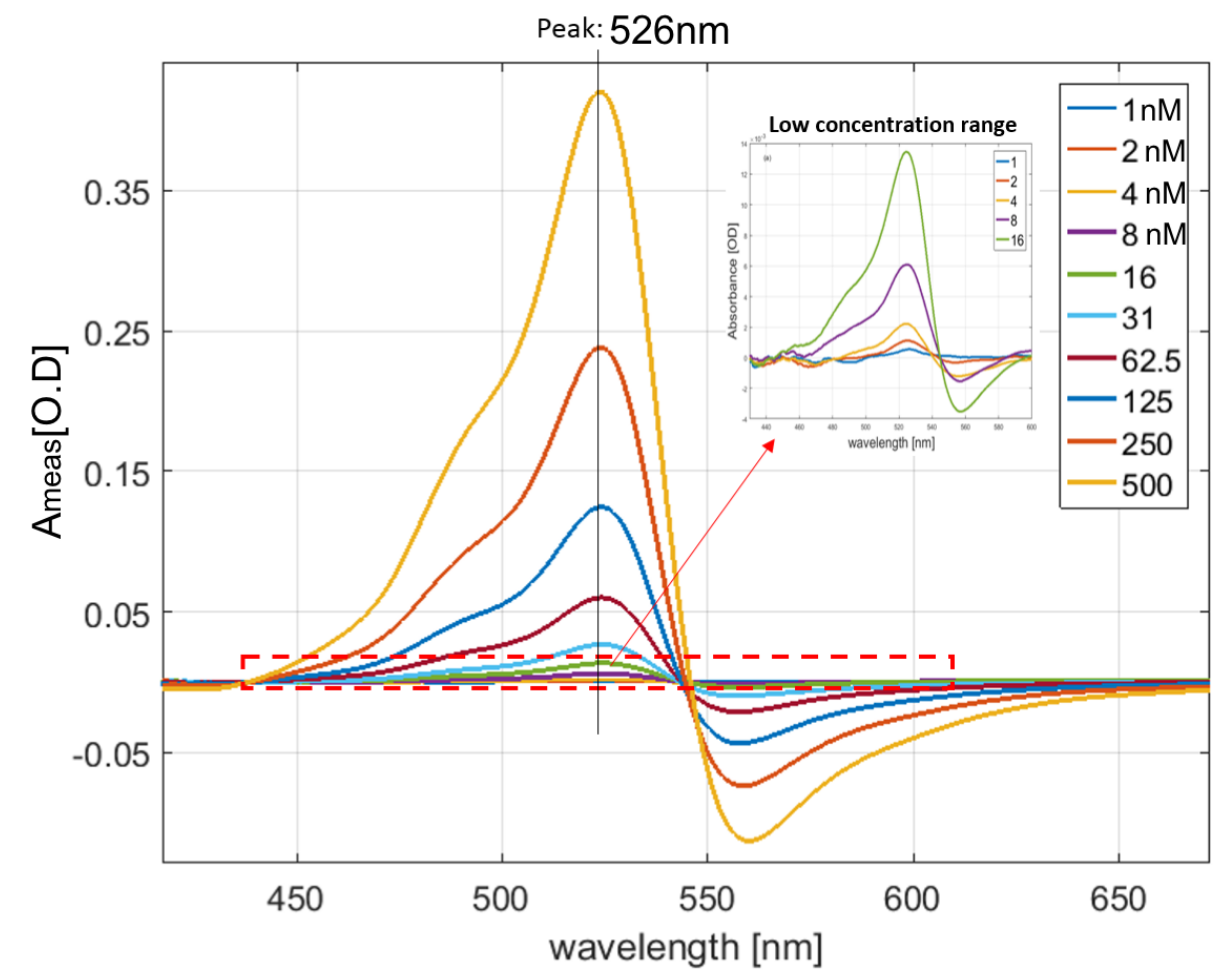

Figure 2.6: The concentration-dependent absorption spectra of R6G in water solution. The concentration of dye varies from $1 \mathrm{nM}$ to $500 \mathrm{nM}$. Notice of the quality of the spectra down to $1 \mathrm{nM}$. 


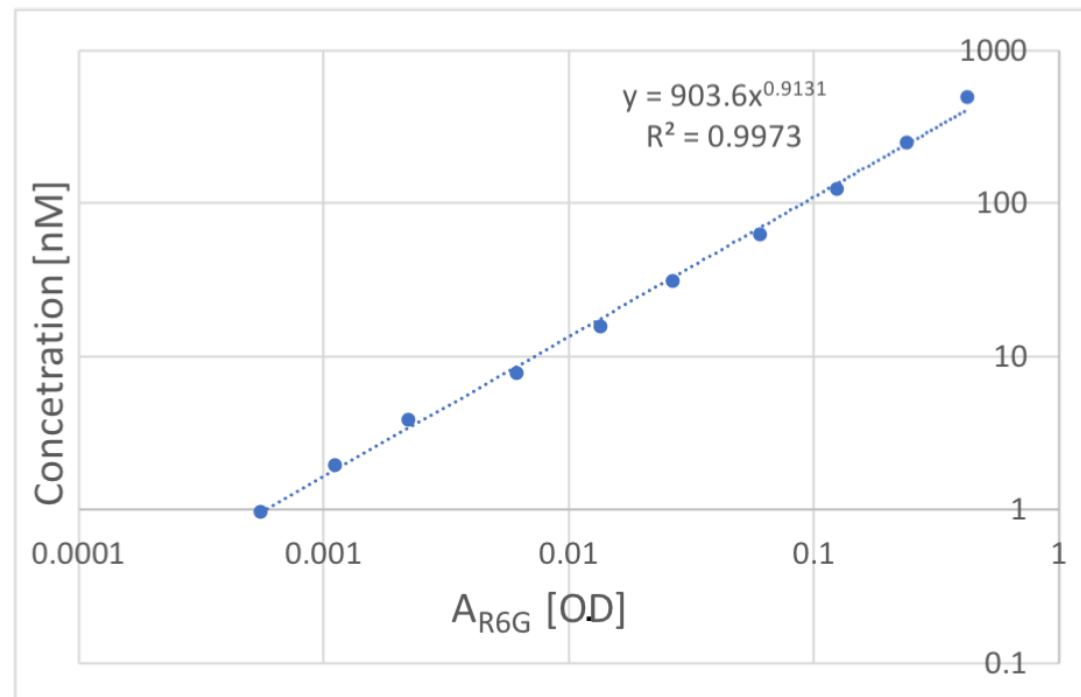

Figure 2.7: Calibration of the integrating sphere absorbance for R6G in water. The relation of the maximum absorbance (at $526 \mathrm{~nm}$ ) and $R 6 G$ concentration is shown in a logarithmic coordinate system. The experimental data (Blue points) $\left(A_{R 6 G}\right.$, Concentration) are fitted well with an exponential function. 


\section{Chapter 3}

\section{Adsorption properties of molecules on NPs}

The adsorption of dyes to the metallic nanoparticle surface is the precondition for the enhancement to happen. A careful assessment of the adsorbed dyes not only is necessary for the deduction of enhancement factor but also provide an insight into the exact mechanism behind the enhanced signal.

In many studies that require a low concentration of adsorbate to avoid the formation of J-aggregates and multilayer on the particles, the adsorption quantification can be difficult and prone to error. A common approach for dyes involves centrifugation of the colloidal solution, followed by a fluorescence or absorption measurement of the supernatant to quantify the concentration of non-adsorbed molecules. We here demonstrate explicitly that this method results in large mistakes in some situations.

We focus specifically on the much-studied case of Rhodamine 6G (R6G) 
adsorbed on silver nanoparticle (AgNP). We show that the type of container used during centrifugation has a dramatic influence on the conclusions, and the discrepancies are related to adsorption of R6G onto the container wall. With a careful choice of container and experimental procedure, we were able to measure the adsorption isotherm of R6G on AgNP at low concentration (down to $\mathrm{nM}$ ). It is notable that for the dye concentrations and the type of nanoparticles we used here, aggregations of dyes and colloids are not expected and for the convenience of discussion, we assume the effects of dyes/colloids aggregations are negligible. Dye aggregation may nevertheless still be possible on the nanoparticle surface.

\subsection{Experimental method}

\subsubsection{Sample preparation}

The low concentrate R6G solutions were prepared from the $100 \mu \mathrm{M}$ stock solution by successive dilutions by factors of 10 to obtain final concentrations from $5 \mathrm{nM}$ to $500 \mathrm{nM}$. The colloidal silver nanoparticles with $\phi 60 \mathrm{~nm}$ silver nanospheres was initially stabilized in the citrate solution, and it is then activated by $1 \mathrm{mM}$ halide solutions ( $\mathrm{KCl}, \mathrm{KBr}$, and $\mathrm{KI}$ ) before mixing with the R6G solution. The mixed samples were always left in vessels for 5 minutes to establish adsorption equilibrium. After that, a Bench-top microcentrifuge was applied to separate the metal-adsorbed molecules from the volume. The supernatant was finally taken for absorption measurement in an integrating cavity (see schematic in Fig.3.1)

The silver colloid was purchased from nanoComposix. R6G (99\% purification) and halide salts were purchased from Sigma Aldrich. 


\subsubsection{Absorption measurement}

The concentration of R6G dyes in the supernatant was estimated from its corresponding absorbance with the equation shown in Fig. 2.7 (chapter 2). The schematic of the adsorption measurement on the R6G-colloid sample is shown in Fig.3.1. The supernatant obtained after centrifugation was taken for absorbance measurement in the integrating sphere. The absorbance is calculated with equation 2.3, where the $I_{\text {reference }}$ and $I_{\text {sample }}$ are the intensities of the collected light for the supernatant solution containing R6G dyes and pure water.

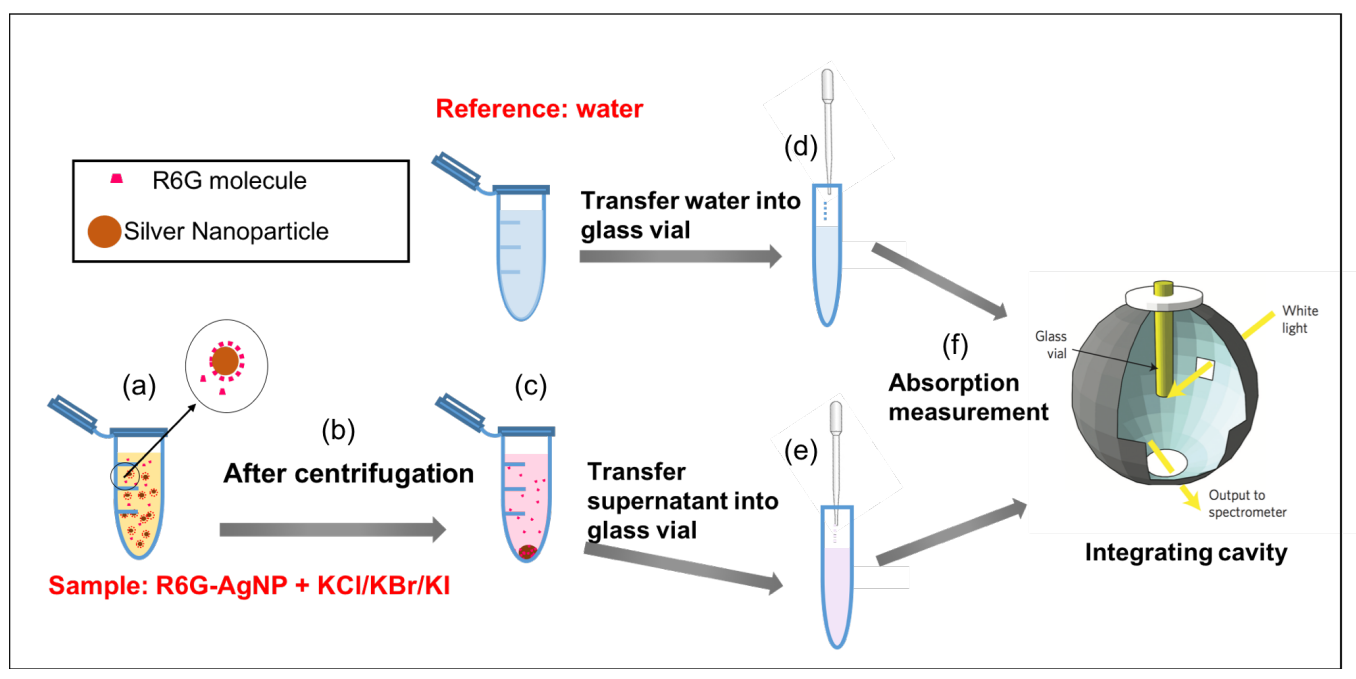

Figure 3.1: Schematic of R6G adsorption quantification. (a) Mixing dyes with halide salts and AgNP. (b) Centrifuging for 10mins at the speed of $14500 \mathrm{rpm}$. (c) The sample after centrifugation is separated into a transparent supernatant phase and a settlement phase. (d) (f) Measuring absorbance of water (reference). (e) (f)Taking the supernatant from sample, and measuring its absorbance. 


\subsection{Results and discussions}

\subsubsection{Unneutral molecules wall adsorption}

When considering the adsorption process in a system that contains unneutral molecules, one should carefully take the vessel wall-adsorption into account. The adsorption competition between the vessel wall and the adsorbent would have a substantial influence on the adsorption outcome and limits the maximum packing density of molecules.

Peter Hildebrandt has reported the phenomenon of the migration of R6G to the vessel walls [27]: for a bare $1 \mathrm{nM}$ R6G solution, they observed a $30 \%$ to $70 \%$ retention of dyes depending on the pretreatment on the vessel. This may also happen in our case and misleads the quantification. To verify this, we examined the concentration of R6G dyes in a wide range of commonly used vessels in the lab. Surprisingly, it turns out that the concentration was also different in the top and the bottom parts of the eppendorf for some commonly used microcentrifuge tubes, which we also attribute to wall adsorption effects.

Table 3.1 summarizes our result. The local concentration distributions of $50 \mathrm{nM}$ R6G solution in various vessels were evaluated, and the effect of centrifuging on the distribution was also tested.

The plain Eppendorf is a standard type of microcentrifuge tube made with Polypropylene, while the Lo-Bind Eppendorfs are known as the type of protein or DNA low retention Eppendorfs and differentiated by a twocomponent polymer modified inner surface [28]. Among the three Eppendorfs, the plain one shows an 'incompatibility' to R6G molecules, since there is twice the amount of R6G on top (31\%) compared to the bottom 


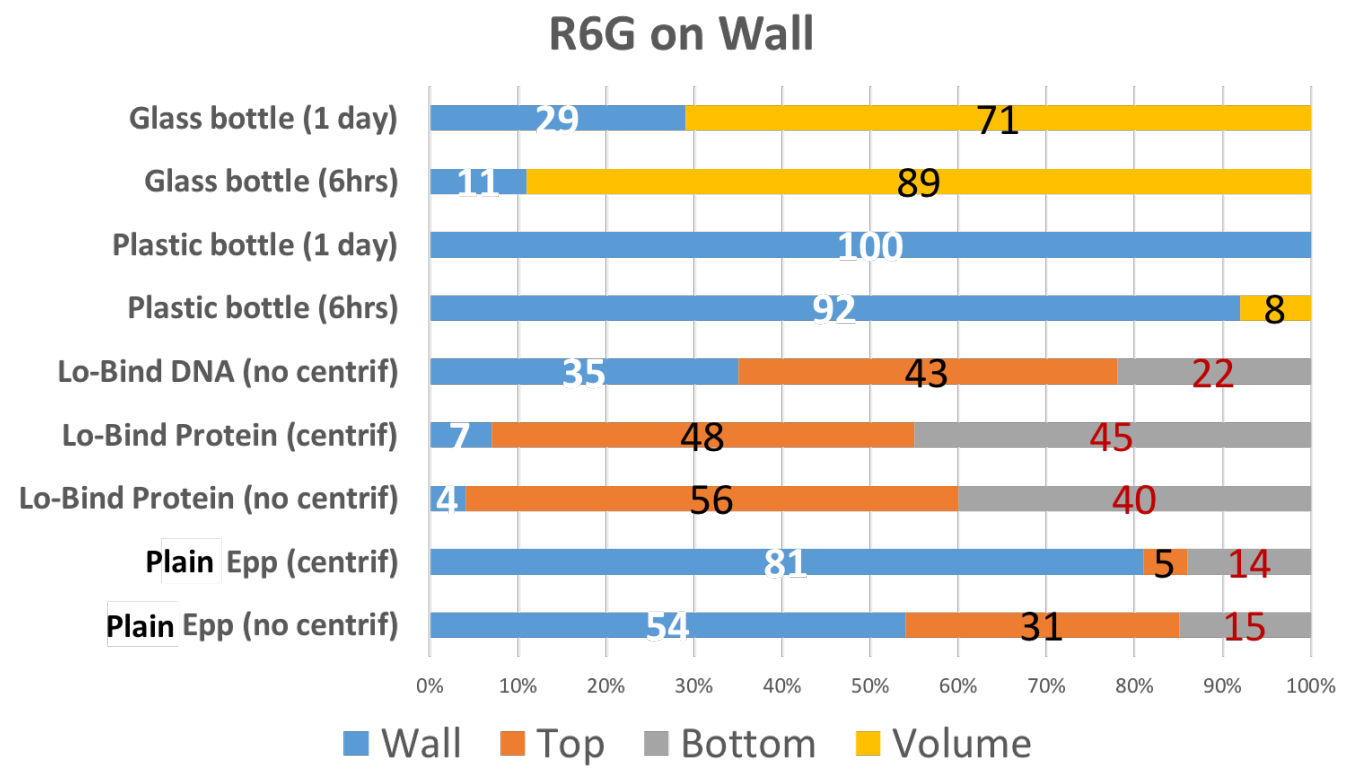

Table 3.1: The local percentage composition of $50 \mathrm{nM}$ R6G solution in various bottles and Eppendorfs. The solution in the latter cases were also centrifuged. The concentration was estimated both from the $1 \mathrm{~mL}$ solution taken from the top and the $1 \mathrm{~mL}$ left bottom half of the container. Note that for glass and plastic bottles, the 'Volume' value is the sum of 'Top' and 'Bottom'.

(15\%), and over half of molecules end up on the wall (54\%). The situation even gets worse after centrifuging for 10 minutes: with $81 \%$ of R6G ending up on the container wall. Contrasting with the normal one, the protein LoBind Eppendorf presents small retention of dyes on the wall, regardless of whether it is centrifuged or not. On the contrary to the plain type, the centrifugal force is instead beneficial for thorough mixing in the Protein Lo-Bind Eppendorf, as indicated by the smaller concentration difference across space. It is also observed that the dyes have a strong tendency of migrating to walls in both glass and plastic bottle: with $29 \%$ and $100 \%$ adsorption after one day. 
A possible explanation for the uneven distribution in some vessels is the positive surface charge of R6G. To verify this, we used eosin, an electrically negative dye molecule to repeat the measurement in the plain Eppendorf. It turned out that it has equal concentrations over the Eppendorf. This verifies the conjecture that the positive charge in R6G, probably together with the negative surface charge of the wall, results in the inhomogeneity in solution. The exact reasons why this happens has not yet been elucidated.

\subsubsection{Adsorption of R6G to Silver Nanoparticles}

Centrifugation provides a straightforward way to quantify the adsorption of molecules in solid suspensions. By applying the centrifugal force, the adsorbed molecules on the solid can be separated from those suspended in the liquid phase. The liquid supernatant is taken for further quantitative analysis using techniques such as absorption and fluorescence spectroscopy. The adsorbed amount is then inferred from the supernatant measurement by taking the difference: $\mathrm{c}_{\text {Adsorption }}=\mathrm{c}_{\text {Total }}-\mathrm{c}_{\text {Supernatant }}$.

One specific case we studied is the adsorption of R6G on silver nanospheres in the presence of various halide anions. Halides $\left(\mathrm{Cl}^{-}, \mathrm{Br}^{-}\right.$, and $\left.\mathrm{I}^{-}\right)$have good affinities to silver and tend to form a negatively charged coating on the silver. Driven by the electrostatic force, the positively charged R6G molecules are attracted and attach to the silver surface within a short time scale

The adsorption with halide anions was evaluated with three typical vessels: the plain Eppendorf, the Protein Lo-Bind Eppendorf, and the Plastic Bottle. Centrifugation was applied to samples in the plain and Lo-Bind Eppendorfs, as illustrated in Fig.3.1. The plastic bottle has the interesting 
property that all the R6G ends up on the wall (table 3.1). So, besides the method of centrifugation, we can utilize the plastic bottle to separate nonAgNP-adsorbed molecules from the bulk phase, as they all adsorbed on the wall.

Figure 3.2 schematically presents the quantifying method by using a plastic bottle. Typically, a long time (6 hrs or longer) is required so that all suspended dyes end up on the container wall. We can then flush the dyes off the wall with ethanol and deduce how much R6G was suspended in the volume (non-adsorbed), and hence by difference the metal-adsorbed amount. For convenience, we denote this approach as 'displacement' method (Fig. 3.2).

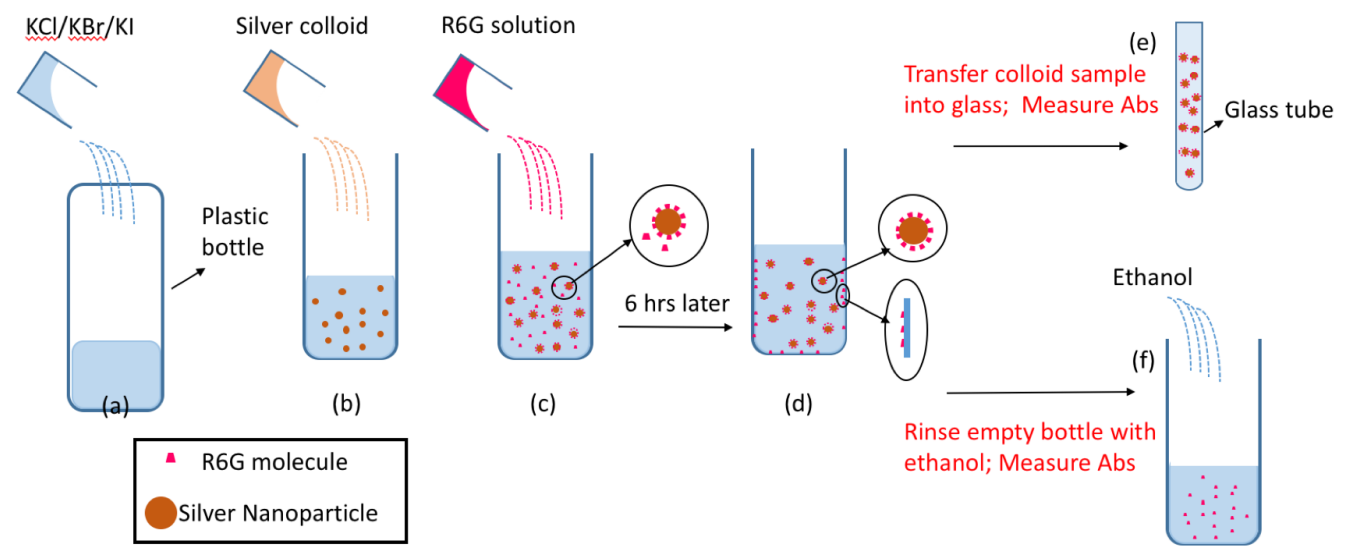

Figure 3.2: Schematic of quantifying the adsorption of R6G on NP using the displacement method. Step (a), (b), (c): mixing the dyes and halides-coated silver colloid. (d): Waiting for 6 hrs until all the non-adsorbed dyes have been 'captured' by plastic bottle walls. (f): Using ethanol to rinse empty bottle. The dyes on wall should be all flushed with ethanol.

Table 3.2 summarizes the adsorbed Rhodamine concentrations measured with the two methods: centrifugation or displacement. In partic- 
ular, the adsorptions of dyes in different halide solutions $(\mathrm{KCl}, \mathrm{KBr}$ and $\mathrm{KI}$ ) were evaluated. For situations of high adsorptions (i.e in $\mathrm{KI}$ and $\mathrm{KBr}$ ), the Plain and the Lo-Bind Protein Eppendorfs give similar results, where the dyes show the largest adsorption in KI: with a $100 \%$ adsorption in both Eppendorfs. The adsorption of R6G is slightly lower in KBr: with 98\% adsorbed in the Plain and the Lo-Bind Protein Eppendorfs, and 84\% adsorbed in the Plastic Bottle. However, for low adsorption cases (i.e in $\mathrm{KCl}$ or without salts), the measured results by different Eppendorfs differ greatly from each other. For $\mathrm{KCl}$ case, we notice an $88 \%$ adsorption in the plain Eppendorf, and 49.2\% adsorption in Lo-Bind Eppendorf. The same sample has been measured using the 'displacement' method as reference: with a 55\% adsorption in plastic bottle. For bare R6G in the Plain tube, we have noticed a large wall retention (table 3.1). This is very likely to happen in the colloids as well, where some suspended dyes migrate to the walls from the supernatant. As a result, the adsorption calculated from the supernatant appears higher than it is because it does not take into account all the dyes that adsorbed to the wall.

Overall, from the results, we conclude that the distribution and adsorption of molecules in suspensions strongly depend on the vessels that used for centrifuging. Especially for studies using charged molecules, we strongly recommend using containers with specialized coating or pretreatment to avoid large wall migration. Based on the above experiment results, we select the Protein Lo-Bind Eppendorf in our subsequent experiments, as it gives the best tradeoff between the accuracy and experiment time. 
Dyes on nanoparticle

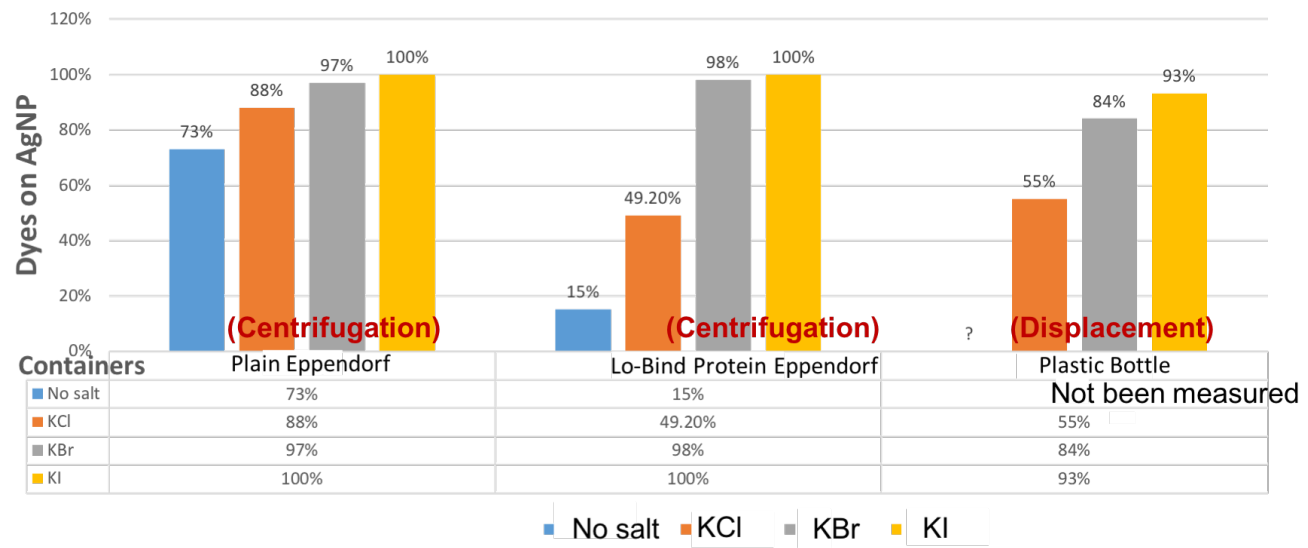

Table 3.2: Adsorption of R6G on silver nanoparticle with and without the presence of $\mathrm{KCl}, \mathrm{KBr}$ and $\mathrm{KI}$ measured in various vessels. Initial concentrations of $\mathrm{R} 6 \mathrm{G}$, AgNP, and salt are $50 \mathrm{nM}, 8 \mathrm{pM}$ and $1 \mathrm{mM}$ respectively.

\subsubsection{Adsorption isotherms in halide solutions}

Adsorption isotherm expresses the amount of molecules adsorbed by the adsorbent at equilibrium state. In our case, this equilibrium would be achieved very fast due to the electrostatic attraction by the anions-coated AgNPs. Typically, the mixtures were left for five minutes to establish the adsorption equilibrium before centrifugation. The adsorption isotherms were measured as a function of dyes concentration, varying from $5 \mathrm{nM}$ to $300 \mathrm{nM}$.

Protein Lo-bind Eppendorf Tubes were used for measuring the isotherms during the whole preparation and centrifugation processes. The concentration of supernatant $C_{\text {free }}$ was obtained by measuring its absorbance in the integrating cavity as described in the chapter 2. 
The key adsorption parameters are described in the Langmuir isotherm:

$$
P=P_{\max } \cdot \frac{K \cdot C_{\text {free }}}{1+K \cdot C_{\text {free }}}
$$

where $\mathrm{P}$ [molecules $\left./ \mathrm{nm}^{2}\right]$ and $P_{\max }\left[\right.$ molecules $\left./ \mathrm{nm}^{2}\right]$ are the packing densities at the equilibrium and maximum. $\mathrm{K}$ is the adsorption constant $\left[n M^{-1}\right]$; $C_{\text {free }}$ is the concentration of free (non-adsorbed) adsorbate species. Langmuir expression is widely applied to describe the monolayer adsorption processes in which the adsorbates have equal chance to be adsorbed to every single site on the adsorbent. In this case, we exclude the situation that dimers forms, as in the low-concentration range, dyes should be isolated from each other.

Knowing the exact concentration of supernatant $C_{\text {free, }}$, we can calculate the packing density of adsorbed R6G by subtracting the R6G in supernatant from the total number. We conduct concentration-dependent experiments to collect the $\left(\mathrm{P}, \mathrm{C}_{\text {free }}\right)$ points, and fit them to Langmuir equation by adjusting the value of the maximum packing density $P_{\max }$ and the adsorption constant $\mathrm{K}$.

Figure 3.3 shows the adsorption isotherms in the three salts and their key parameters are summarized in Table 3.3. Over the whole concentration range, which corresponds to a submonolayer to the monolayer coverage, the addition of KI (fig 3.3 (c)) promotes the adsorption process most: with the adsorption constant of $0.904 n M^{-1}$. This is 25 times larger than the case of $\mathrm{KCl}\left(0.00623 n M^{-1}\right)$, and about 5.6 times larger than the case of $\mathrm{KBr}\left(0.160 n \mathrm{M}^{-1}\right)$. This explains why R6G has the least adsorption in $\mathrm{KCl}$ and the largest adsorption in $\mathrm{KI}$ at $50 \mathrm{nM}$ (as shown in Table.3.2). The packing capacities $\left(P_{\max }\right)$ of silver in the three cases are similar: with the value of $1.27,1.47$ and 1.08 molecules $/ \mathrm{nm}^{2}$ for $\mathrm{KCl}, \mathrm{KBr}$ and $\mathrm{KI}$ respectively. 

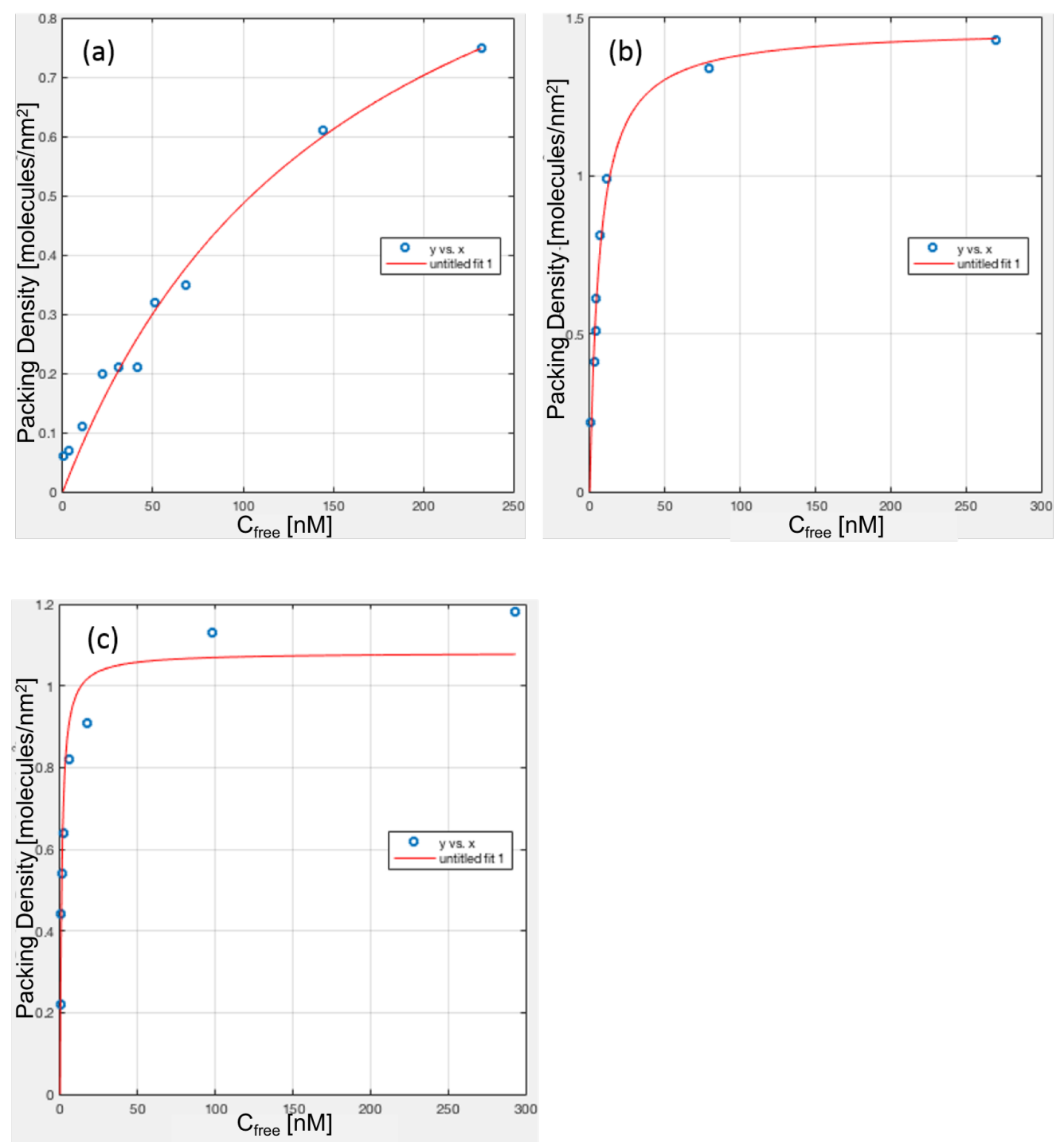

Figure 3.3: Adsorption isotherm of R6G on silver nanoparticles with the addition of $\mathrm{KCl}(a), \mathrm{KBr}(b)$, and $\mathrm{KI}(\mathrm{c})$ : circle points are experimental data. The total dye concentration $\left(C_{\text {free }}\right)$ ranges from $5 \mathrm{nM}$ to $300 \mathrm{nM}$. Experimental data are further fitted with Langmuir relation (see eq.3.1).

Knowing the adsorption constant $\mathrm{K}$, we can further calculate the ad- 


\begin{tabular}{|l|l|l|}
\hline & $P_{\max }\left[\right.$ molecule $\left./ \mathrm{nm}^{2}\right]$ & $K\left[n M^{-1}\right]$ \\
\hline $\mathrm{KCl}$ & 1.27 & 0.00623 \\
\hline $\mathrm{KBr}$ & 1.47 & 0.160 \\
\hline $\mathrm{KI}$ & 1.08 & 0.904 \\
\hline
\end{tabular}

Table 3.3: $P_{\max }$ and $K$ are simulated with Langmuir equation. Results are with confidences larger than 95\%.

sorption energy $(\Delta G)$ with the related equation:

$$
\Delta G=-R T \ln (K)
$$

The $\Delta G$ are $-47.1 \mathrm{~kJ} / \mathrm{mol},-54.8 \mathrm{~kJ} / \mathrm{mol}$ and $-59.1 \mathrm{~kJ} / \mathrm{mol}$ for $\mathrm{KCl}, \mathrm{KBr}$, and $\mathrm{KI}$ at room temperature (i.e. $15^{\circ} \mathrm{C}$ ). These energies are much higher than what is expected in the physisorption process driven by van der Waals force (i.e. approximatly $20 \mathrm{~kJ} / \mathrm{mol}$ ) [29]. This implies that the halide activated R6G adsorption is a chemisorption process. This observation is consistent with the previous findings in [27]. In this paper, Peter Hildebrandt proposed one possibility that the positively charged R6G molecules bind to the silver surface through $\mathrm{N}^{+}-\mathrm{Cl}^{-}-\mathrm{Ag}^{+}$covalent bond. Rather than orient flatly, R6G molecules are more likely tilted with an angle on the sphere via N-Ag bonds.

Another interesting finding is that R6G has such different affinities to silver in the presence of various salts. The fact that R6G has the largest adsorption in $\mathrm{KI}$ and least adsorption in $\mathrm{KCl}$ is different from our expectation in a plasmonics or SERS context. Because normally, the optical response of R6G has the least enhancement in $\mathrm{KI}$, but the largest enhancement in $\mathrm{KCl}[30]$. This, however to some extent, confirms that it is not the packing density, but other factors, such as orientation and dyes interaction that contribute to the enhancement and spectral change. In the subsequent chapter we will give more discussion regarding this. 


\section{Chapter 4}

\section{Modified absorbance of R6G on silver}

Another focus of this project is understanding the origins of the modified absorption of molecules on metallic nanoparticles. This is studied by measuring the differential absorption of dyes at submonolayer coverage, where the dye-NP and dye-dye interaction effects on the absorption modifications can be differentiated. Specifically, we focus on one problem: the electromagnetic and chemical effects involved in the spectral modification of R6G dyes adsorbed to AgNPs in the presence of halide ions. 


\subsection{Experimental method}

\subsubsection{Surface absorbance measurement in the integrating cavity}

Figure 4.1 shows the basic idea to obtain the surface absorbance of dyes on nanoparticles. A systematic process consists of measuring the transmitted light for both NP+dye and bare NP colloid sample. The differential absorbance of dyes is then retrieved by subtracting the reference (bare $\mathrm{NP})$ spectrum from sample (NP+dye) spectrum (see the yellow curve on Fig.4.1), which can also be calculated by equation below:

$$
A=-\log \frac{I_{N P+d y e}}{I_{N P}}
$$

where $I_{N P+d y e}$ is the intensity of transmitted light through the (NP+dye) sample, and $I_{N P}$ is the intensity of transmitted light through the bare NP sample. Note that the intensities, as well as absorbance, are functions of wavelength. The real absorbance $\left[\mathrm{cm}^{-1}\right]$ can then be obtained by correcting with the effective path length $\mathrm{L}[\mathrm{cm}]$ as discussed in chapter 2.

\subsection{Results and discussions}

\subsubsection{Low-coverage surface absorption}

To study the intrinsic effect of the nanoparticle on the R6G optical response, we need to access very low dye coverage where the dye-dye interactions are negligible and have little effect on the final absorption spectra. 


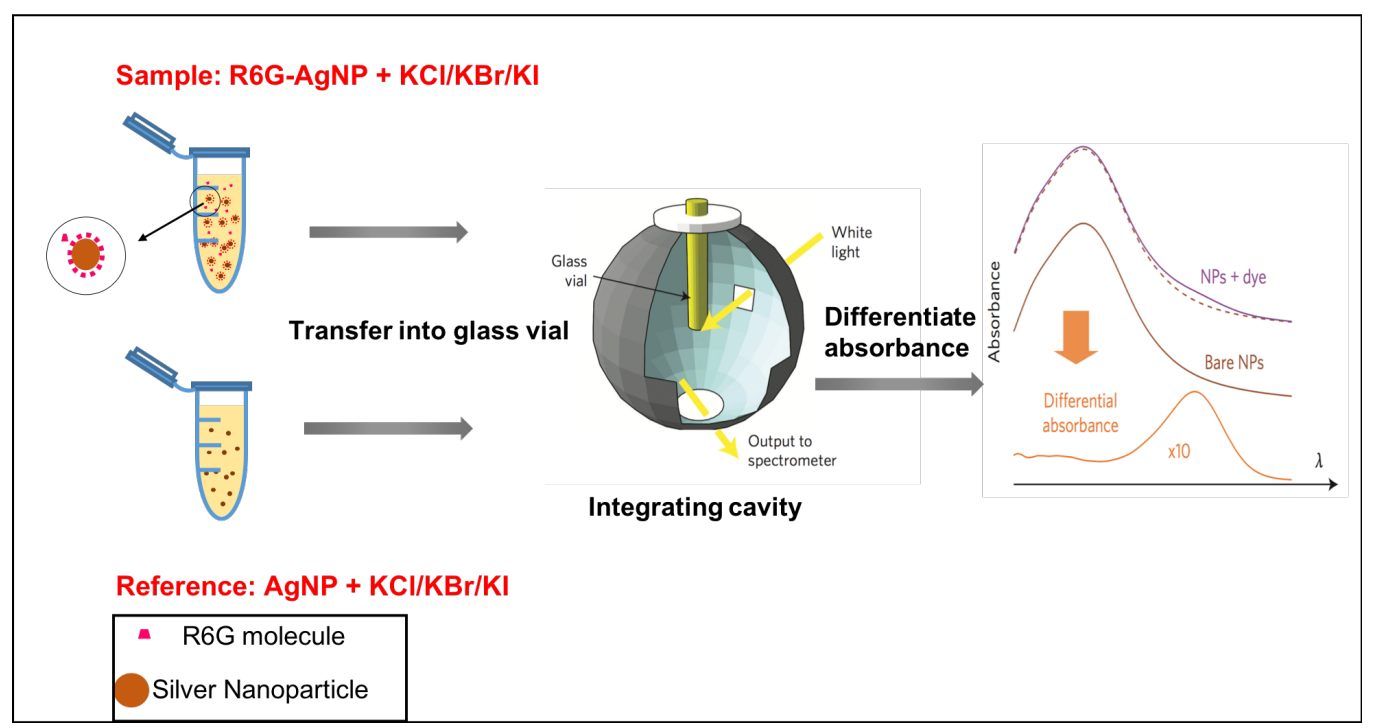

Figure 4.1: Schematic of a differential absorption measurement.

By using the integrating cavity, we are able to measure $5 \mathrm{nM}$ R6G in colloid sample, which corresponds to a surface coverage of $0.05 \mathrm{dye} / \mathrm{nm}^{2}$. This should be a safe concentration to avoid dye-dye interactions.

Figure 4.2 shows the modified absorption spectra of R6G on silver nanoparticle. The spectra at low concentration are red-shifted by different extent depending on the activating anions: with a $13 \mathrm{~nm}$ redshift for $\mathrm{KCl}, 18 \mathrm{~nm}$ redshift for $\mathrm{KBr}$, and $22 \mathrm{~nm}$ redshift for $\mathrm{KI}$ compared to the original absorption of R6G in water (dash line), which peaks at $526 \mathrm{~nm}$. Since there is no dye-dye interaction, one may instead explain the spectral shift with the electromagnetic self-reflected field (image dipole effect). But this has been proved not to be the case in [22]: artificially adjusting the dipole-sphere distance to $0.5 \mathrm{~nm}$ to match the observed spectral shift of the main peak does not reproduce well the spectral line shape. An alternative explanation of the spectral shift is the chemical change of R6G molecule, that is, the modification of electronic structure by the silver surface and halide anions. As the results in chapter 3.2.3 indicate, the halide activated 
R6G adsorption is a chemisorption process, during which the positively charged dyes likely bind to the silver surface through $\mathrm{N}^{+}-\mathrm{Cl}^{-}-\mathrm{Ag}^{+}$covalent bond, causing an internal electrons reconfiguration and therefore a change in the polarizability.

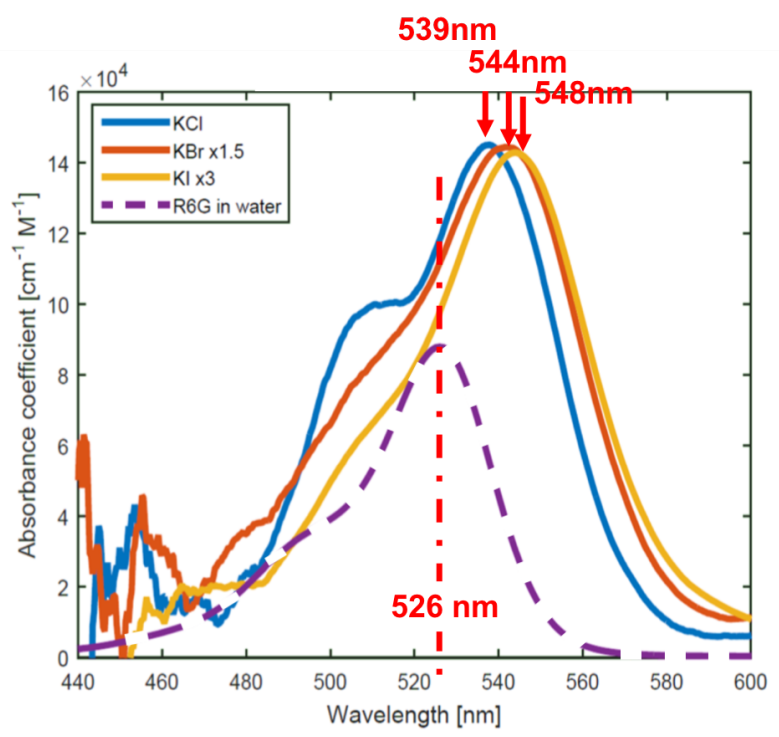

Figure 4.2: Surface absorption spectra of $5 n M$ R6G in silver colloid in the presence of $\mathrm{KCl}$ (blue plot), $\mathrm{KBr}$ (red plot) and $\mathrm{KI}$ (yellow plot), referenced against R6G in water (dashed purple plot). Plots are normalized for the convenience of comparison.

One can also observe an enhancement or quenching in the absorption intensities: with a factor the order of 2 for $\mathrm{KCl}, 1$ for $\mathrm{KBr}$ and 0.7 for KI. The difference in the magnitude could be explained by the different tilted angles of the dyes on the sphere. According to the theoretical modelling in [22], the orientation of a dipole decides how much it is subjected to the electromagnetic field enhancement created by the sphere: radially-oriented dipoles exhibit a much larger enhancement than those tangentially-oriented dipoles. Comparing with our experimental results, it is reasonable to propose that the halide-induced dipole tilted angle (with 
respect to the tangent) follows an order of $\mathrm{KCl}>\mathrm{KBr}>\mathrm{KI}$. However, we note here that the Surface Enhanced Raman (SERS) response of R6G in silver colloid shows the largest enhancement in $\mathrm{KBr}$ instead of $\mathrm{KCl}$. This disagreement might be attributed to the different aggregation state of the colloid: $\mathrm{Br}^{-}$tends to aggregate colloids whereas the low $\mathrm{Cl}^{-}$concentration does not induce any aggregation in our experiments. The silver clusters formed in the former case are of an advantage to create hot spots, and thus produce an intense local field enhancement.

\subsubsection{Concentration-dependent surface absorption}

For many studies that apply plasmon/dye interaction, the concentration of adsorbed molecules need to be sufficiently high to achieve a detectable signal. The absorption spectrum of the dyes in such high coverage situation is therefore being influenced significantly by the dye-dye interactions (i.e electromagnetic coupling between dyes). Experimentally, this dipole-dipole interaction was studied by varying the concentration of surface dyes. The concentration of R6G varies from $5 \mathrm{nM}$ to $100 \mathrm{nM}$, corresponding to the surface coverage between 0.05 to 1 molecule $/ \mathrm{nm}^{2}$. The concentration-dependent absorption results are shown in Fig. 4.3. The raw absorption spectra (panel $(\mathrm{a})(\mathrm{b})(\mathrm{c})$ ) are also normalized with the number of dyes for the convenience of comparison (panel (d)(e)(f)).

When considering of a simple dimer system consisting of two dipoles, the effect of one dipole on the other would cause a spectral shift depending on the relative distance and orientations of these two dipoles: typically a blue-shift for H-aggregates (side by side coupling) and a red-shift for Jaggregates (head to tail coupling) [22]. At decreasing separations, their electromagnetic interactions become stronger, resulting in further spectral 

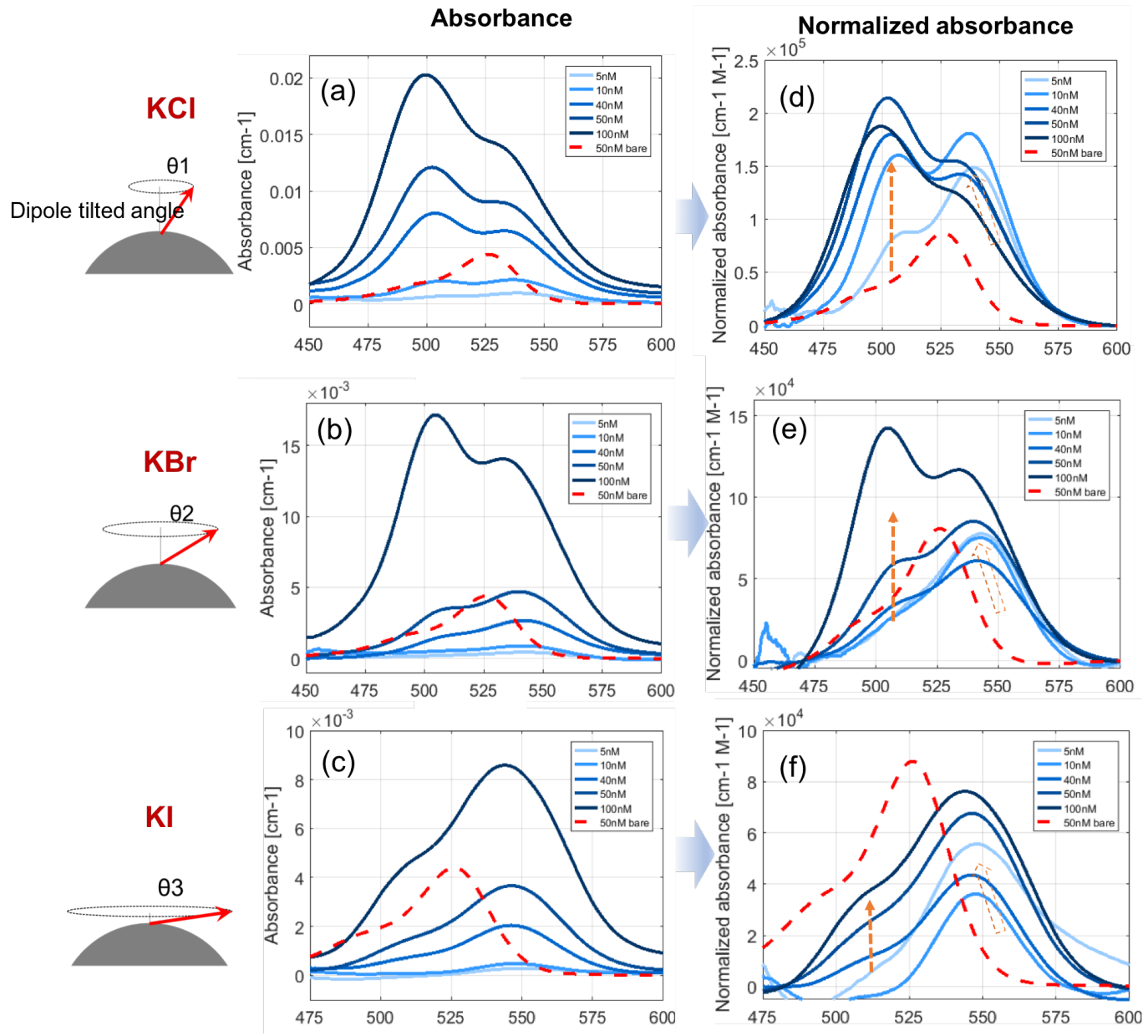

Figure 4.3: Surface absorption spectra of R6G, with concentrations varied from $5 \mathrm{nM}$ to $100 \mathrm{nM}$, in $\mathrm{KCl}(a), \mathrm{KBr}(b)$ and $\mathrm{KI}(\mathrm{c})$. Normalized surface absorption spectra of $\mathrm{R} 6 \mathrm{G}$ in $\mathrm{KCl}(d), \mathrm{KBr}(e)$ and $\mathrm{KI}(f)$

shifts. On a metal surface we expect J-aggregates type interaction if the dyes are adsorbed flat (in plane) on the surface and H-aggregates type if they are upright or even slightly tilted, because the plasmonics enhancement is much larger for the perpendicular component.

From panel (d) - (f) in Fig. 4.3, the main peaks in all three cases show 
tendencies of blue-shift at increasing dye concentration. This confirms the intepretation that the dyes are tilted with an angle on the silver surface, producing a partly side-by-side (H-aggregates liked) interaction between neighbouring dipoles.

We also observe a clear increase in the relative intensity of the shortwavelength absorption peak at increasing dye concentration. This increase of the intensity follows the order of $\mathrm{KCl}>\mathrm{KBr}>\mathrm{KI}$ and can be attributed to the strong dye-dye interaction. One natural explanation for the differences is that the dyes adsorbed the most in $\mathrm{KCl}$, and least in $\mathrm{KI}$. As predicted in [22], the shoulder peak goes higher with more dyes on silver. But this is not the case because our findings in chapter 3 show that R6G adsorbs the most in the presence of $\mathrm{I}^{-}$rather than $\mathrm{Cl}^{-}$. An alternative explanation is the tilt angle difference, i.e. a modification of anions induced dipole orientations. Such orientation induced spectral changes can be well predicted by the electromagnetic model recently developed by our group [22]. It theoretically shows that the growth of the shoulder peak strongly depends on the dipoles tilted angle: with a higher peak for dyes that are sitting more upright on the particle, and a weaker peak for dyes that more tangential to the sphere. By comparing it with our experimental results we can deduct that R6G molecules lay more vertically on the sphere in $\mathrm{KCl}$ as it has the strongest shoulder peak, and more flatly in KI. This intepretation would also explain the fact that the dyes on the $\mathrm{Cl}^{-}$-coated AgNP exhibit the largest enhancement factor and the least enhancement in KI (Fig.4.2).

\subsubsection{Could it be chemical etching?}

So far our interpretation regarding the spectral modifications was more focus on the electromagnetic interaction effects: dye-dye coupling, and 
dye-NP orientation effect. The latter is considered to have a close relation with the presence of halide anions, which we believe help connect the dyes to the sphere through a strong $\mathrm{N}^{+}-\mathrm{Cl}^{-}-\mathrm{Ag}^{+}$covalent bond. The exact mechanism has not been evidenced though, but one thing for sure is that the addition of halide anions largely enhances the intensities of Raman scattering. Such enhancement partly comes from the standard plasmonic induced strong field near sphere, namely the electromagnetic effect. This may also arise from the chemically induced changes of the surface such as surface roughness / sculpturing and adatoms formation [31-33]. The latter applies only for species that can react to the surface, so it cannot explain the observed signal enhancement in all cases.

The system consisting of R6G and colloidal silver in the presence of halide anions has been studied a lot in the context of SERS. However, the exact mechanism behind the enhanced signal in SERS is still a matter of debate in the literature $[21,27,34]$. There are studies indicating that the halide anions $\left(\mathrm{Br}^{-}, \mathrm{Cl}^{-}\right)$can sculpture the corners of silver nanoprisms into nanodisks $[18,32]$. The dissolved silver atoms or ions may further form small clusters, which were proposed to enlarge the signal [33, 35, 36]. These reactions may result in halide-induced roughness, which could affect our interpretations.

To check whether the etching affects our results, we designed simple measurements based on consecutively switching the salt species according to their different affinities to silver. Two salts are tested at once, and the second salt species is added with a time delay (10min) after the first salt, ensuring that the first species has enough time to diffuse to the NP surface and make its effect. The reversibility of the salt effect on the sphere can be checked by the evolution of the absorption spectrum of R6G during the salt switching. The 'switching' results are shown in Fig.4.4. 

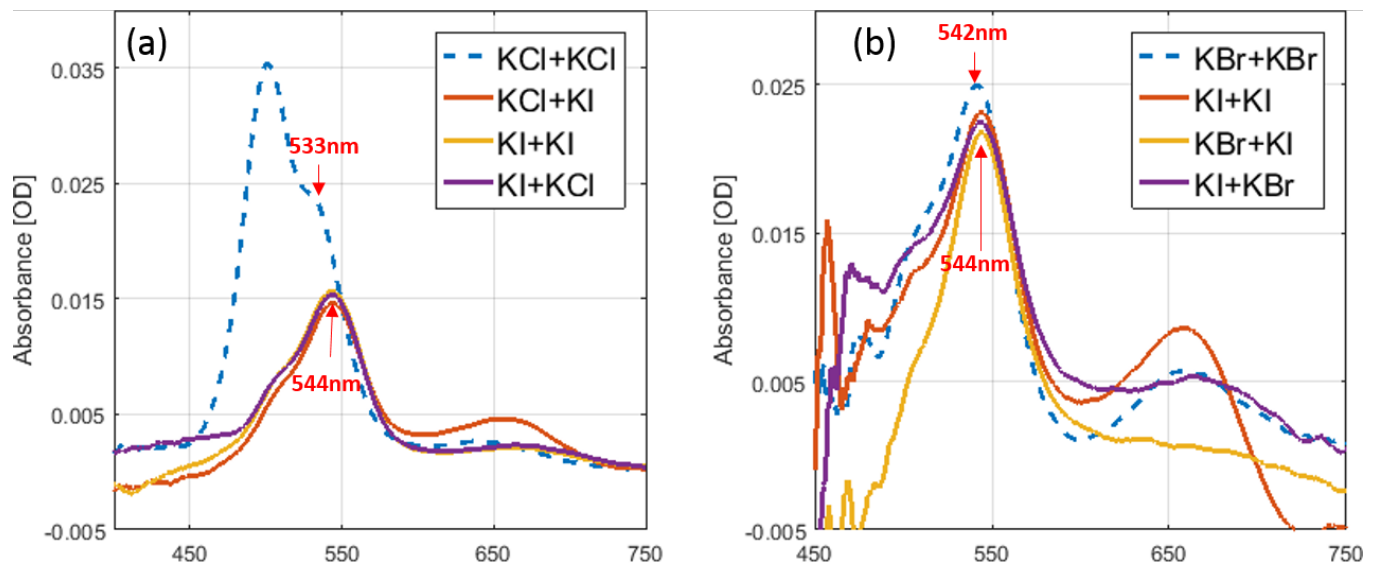

Figure 4.4: Differential absorption spectra of $50 \mathrm{nM}$ R6G in the bi-anions activated colloidal silver: (a) with $\mathrm{KCl}$ and $\mathrm{KI}$ added at different time in a sequence. (b) with $\mathrm{KBr}$ and $\mathrm{KI}$ added at different time in a sequence.

In Fig.4.4 (a), one can see that for samples with KI, regardless of the adding sequence, the absorption spectra all resemble each other, showing a peak at $544 \mathrm{~nm}$. Comparing with the $(\mathrm{KCl}+\mathrm{KCl})$ sample, which peaks at $533 \mathrm{~nm}$, we can infer that the $\mathrm{Cl}^{-}$is always replaced by $\mathrm{I}^{-}$when KI exists, which means KI has a better affinity to the silver surface. This is consistent with the results of chapter 3 and the fact that AgI has lower solubility in water [37]. Moreover, the fact that the spectrum of (KI+KI) overlaps with $(\mathrm{KCl}+\mathrm{KI})$ indicates that the previously added chloride in the later sample does not cause any irreversible change to the sphere, such as etching and roughening.

We can come to similar conclusions from Fig.4.4 (b) that the iodide has better affinities to silver surface compared with bromide, and $\mathrm{KBr}$ does not do any irreversible change to the silver sphere. It should be noted that the peculiar band shown at $650 \mathrm{~nm}$ may due to the silver colloid aggregation during the addition of the second salt. All in all, we can conclude that both the chloride and bromide do not have etching or roughening effect on the 
spherical silver particle or if they do, it does not affect the R6G adsorption spectral intensity or shifts. We can therefore exclude the possibility of roughening-induced spectral changes and be confident with the argument given in the previous sections. 


\section{Chapter 5}

\section{Conclusion}

In this thesis, systematic measurements of the surface absorbance spectra of dyes adsorbed on silver nanoparticle were carried out with an integrating cavity. Our results highlight the great potential of this method for the ultra-low absorbance measurement in the strongly scattering media such as metal colloids. We specifically focus on an R6G and halide anions activated colloidal silver system, which is of important relevance to the studies of plasmonics and surface enhanced spectroscopies.

Our results of the low-coverage absorption measurements indicate that modifications of the intrinsic dye polarizability by silver sphere appear to occur in the presence of halide anions. We believe this 'chemical' modification is related to the formation of the covalent bond $\mathrm{Ag}^{+}-\mathrm{Cl}^{-} / \mathrm{Br}^{-} / \mathrm{I}^{-}-\mathrm{N}^{+}$. More realistic situations where the surface coverage approaches monolayer are also considered and experimentally achieved by a concentrationdependent measurements. Our results indicate that the effect of dye-dye interaction is actually significant on the spectral changes even at relatively low surface coverage. Both of these effects (dye-metal and dye-dye inter- 
action) should be carefully considered for the studies involving adsorbed molecules on metal particles.

We are planning to write up the results of Chapter 4 into a manuscript entitled "Studying adsorbate orientation and molecule/surface interactions using surface absorbance measurements" by Xiaohan Chen, Johan Grand, Brendan L. Darby, Matthias Meyer, Baptiste Auguie, and Eric C. Le $\mathrm{Ru}$, which we will submit to ACS Nano or ACS Photonics. An earlier version of this paper was drafted in the course of my study, but the submission was delayed while the theoretical models to explain the experimental results were developed in parallel (by other members of the group).

Another aspect of the study lays in the quantification of dyes adsorbed on the particle. The quantification of adsorption is of vital importance in the estimation of key parameters such as the enhancement factor of SERS, and the reaction rate of a reaction. We have shown that the selection of vessels for centrifugation and stocking has a significant impact on the quantification results. Especially for unneutral molecules, using an inappropriate container for keeping and separating would cause molecules to accumulate on the wall, and even leads to non-uniform local distribution. Besides that, we measured the adsorption isotherms of R6G in the presence of $\mathrm{KCl}, \mathrm{KBr}$, and $\mathrm{KI}$, and identified the adsorption difference between each case. Up to now, the experimental determination of SERS enhancement factors in such systems had assumed a 100\% adsorption. In view of our results, this assumption should clearly be revisited in the common case of $\mathrm{Cl}^{-}$even at low dye concentration.

The results of Chapter 3 will form the basis for a manuscript entitled "Wall adsorption effects in the centrifugation method for the quantification of adsorption isotherms on nanoparticles" by Xiaohan Chen, Johan 
Grand, Eric C. Le Ru, which we intend to submit to a journal such as Langmuir of Phys. Chem. Chem. Phys. 


\section{Bibliography}

[1] Eric C. Le Ru and Pablo G. Etchegoin. Raman spectroscopy and related optical techniques. In Principles of Surface-Enhanced Raman Spectroscopy, pages 29-120. 2009. ISBN 9780444527790. doi: 10.1016/ b978-0-444-52779-0.00008-8.

[2] Brendan L. Darby, Pablo G. Etchegoin, and Eric C. Le Ru. Singlemolecule surface-enhanced Raman spectroscopy with nanowatt excitation. Physical Chemistry Chemical Physics, 16(43):23895-23899, 2014. ISSN 14639076. doi: 10.1039/c4cp03422h.

[3] Katrin Kneipp, Yang Wang, Harald Kneipp, Lev T. Perelman, Irving Itzkan, Ramachandra R. Dasari, and Michael S. Feld. Single molecule detection using surface-enhanced raman scattering (SERS). Physical Review Letters, 78(9):1667-1670, 1997. ISSN 10797114. doi: 10.1103/ PhysRevLett.78.1667.

[4] Koichi Awazu, Makoto Fujimaki, Carsten Rockstuhl, Junji Tominaga, Hirotaka Murakami, Yoshimichi Ohki, Naoya Yoshida, and Toshiya Watanabe. A plasmonic photocatalyst consisting of silver nanoparticles embedded in titanium dioxide. Journal of the American Chemical Society, 130(5):1676-1680, 2008. ISSN 00027863. doi: 10.1021/ja076503n.

[5] Suljo Linic, Umar Aslam, Calvin Boerigter, and Matthew Morabito. 
Photochemical transformations on plasmonic metal nanoparticles, 2015. ISSN 14764660.

[6] Phillip Christopher, Hongliang Xin, and Suljo Linic. Visible-lightenhanced catalytic oxidation reactions on plasmonic silver nanostructures. Nature Chemistry, 3(6):467-472, 2011. ISSN 17554330. doi: 10.1038/nchem.1032.

[7] Harry A. Atwater and Albert Polman. Plasmonics for improved photovoltaic devices, 2010. ISSN 14761122.

[8] Emmanuel Stratakis and Emmanuel Kymakis. Nanoparticle-based plasmonic organic photovoltaic devices, 2013. ISSN 13697021.

[9] Eric C. Le Ru and Pablo G. Etchegoin. Single-Molecule SurfaceEnhanced Raman Spectroscopy. Annual Review of Physical Chemistry, 63(1):65-87, 2012. ISSN 0066-426X. doi: 10.1146/ annurev-physchem-032511-143757.

[10] Brendan L. Darby, Baptiste Auguié, Matthias Meyer, Andres E. Pantoja, and Eric C. Le Ru. Modified optical absorption of molecules on metallic nanoparticles at sub-monolayer coverage. Nature Photonics, 10(1):40-45, 2016. ISSN 17494893. doi: 10.1038/nphoton.2015.205.

[11] James M. Palmer and Barbara G. Grant. The Art of Radiometry. 2009. doi: $10.1117 / 3.798237$.

[12] Robin M Pope and Edward S Fry. pure water . $\{$ II $\}$. Integrating cavity measurements. Applied Optics, 36:87108723 ST - Absorption spectrum (380 -700 nm), 1997. URL http://www.opticsinfobase.org/abstract. Cfm?URI=ao-36-33-87100P-Ja $\left\{\backslash \frac{0}{0}\right.$ 5Cnhttp: / / www . opticsinfobase.org/abstract. cfm?URI=ao-36-33-8710. 
[13] Edward S. Fry, George W. Kattawar, and Robin M. Pope. Integrating cavity absorption meter. Applied Optics, 31(12):2055, 2009. ISSN 00036935. doi: 10.1364/ao.31.002055.

[14] A.K. Gaigalas, Hua-Jun He, and Lili Wang. Measurement of Absorption and Scattering With an Integrating Sphere Detector: Application to Microalgae. Journal of Research of the National Institute of Standards and Technology, 114(2):69, 2012. ISSN 1044677X. doi: 10.6028/jres.114.006.

[15] Stelvio Tassan and Giovanni M. Ferrari. Variability of light absorption by aquatic particles in the near-infrared spectral region. Applied Optics, 42(24):4802, 2007. ISSN 0003-6935. doi: 10.1364/ao.42.004802.

[16] Rüdiger Röttgers, Clivia Häse, and Roland Doerffer. Determination of the particulate absorption of microalgae using a point-source integrating-cavity absorption meter. Limnology and Oceanography: Methods, 5:1-12, 2007. doi: 10.4319/lom.2007.5.1.

[17] Dariusz Stramski, Sławomir B. Woźniak, and Piotr J. Flatau. Optical properties of Asian mineral dust suspended in seawater. Limnology and Oceanography, 49(3):749-755, 2004. ISSN 00243590. doi: 10.4319/ lo.2004.49.3.0749.

[18] Shih Hong Ciou, Yi Wei Cao, Huai Cing Huang, De Yan Su, and Cheng Liang Huang. SERS enhancement factors studies of silver nanoprism and spherical nanoparticle colloids in the presence of bromide ions. Journal of Physical Chemistry C, 113(22):9520-9525, 2009. ISSN 19327447. doi: 10.1021/jp809687v.

[19] Almar Palonpon, Taro Ichimura, Prabhat Verma, Yasushi Inouye, and Satoshi Kawata. Halide-ion-assisted increase of surface-enhanced hyper-Raman scattering: A clear observation of the chemical effect. 
Journal of Raman Spectroscopy, 40(2):119-120, 2009. ISSN 03770486. doi: $10.1002 /$ jrs.2169.

[20] Garry P. Glaspell, Chen Zuo, and Paul W. Jagodzinski. Surface enhanced raman spectroscopy using silver nanoparticles: The effects of particle size and halide ions on aggregation. Journal of Cluster Science, 16(1):39-51, 2005. ISSN 10407278. doi: 10.1007/s10876-005-2714-x.

[21] Denis Pristinski, Siliu Tan, Melek Erol, Henry Du, and Svetlana Sukhishvili. In situ SERS study of Rhodamine 6G adsorbed on individually immobilized Ag nanoparticles. Journal of Raman Spectroscopy, 37(7):762-770, 2006. ISSN 03770486. doi: 10.1002/jrs.1496.

[22] Baptiste Auguié and Eric C. Le Ru. Optical Absorption of Dye Molecules in a Spherical Shell Geometry. Journal of Physical Chemistry C, 122(33):19110-19115, 2018. ISSN 19327455. doi: 10.1021/acs. jpcc.8b05542.

[23] Labsphere. Technical guide: Integrating sphere radiometry and photometry. 2012. URL https:// wWw. labsphere.com/site/assets/files/2550/ a-guide-to-integrating-sphere-radiometry-and-photometry . pdf.

[24] Labsphere. Technical Guide: Integrating Sphere Theory and Applications. MANUAL for Integration Sphere, pages 1-19, 2011. ISSN 15422224. URL http: / /www. labsphere.com/.

[25] Labsphere. Reflectance Materials and Coatings. pages 1-26, 2014.

[26] G. Bain. Integrating sphere diffuse reflectance technology for use with uv-visible spectrophotometry. Thermo Fisher Scientific, Madison, Wisconsin, 2007. 
[27] Peter Hildebrandt and Manfred Stockhurger. Surface-Enhanced Resonance Raman Spectroscopy of Rhodamine 6G adsorbed on colloidal silver. Journal of Physical Chemistry, 88(24):5935-5944, 1984. ISSN 00223654. doi: 10.1021/j150668a038.

[28] Protein lobind tubes. 2019. URL https:

//online-shop.eppendorf.com.au/AU-en/

Laboratory-Consumables-44512/Tubes-44515/

Protein-LoBind-Tubes-PF-56251.html?_ga=2 .

225781855.452649609.1553655074-938121821.

1525396002. Accessed: 2019-03-27.

[29] Carlos P Bergmann and Fernando Machado Machado. Carbon nanomaterials as adsorbents for environmental and biological applications. Springer, 2015.

[30] M. Futamata and Y. Maruyama. LSP spectral changes correlating with SERS activation and quenching for R6G on immobilized Ag nanoparticles. Applied Physics B: Lasers and Optics, 93(1):117-130, 2008. ISSN 09462171. doi: 10.1007/s00340-008-3179-z.

[31] H. Wetzel, H. Gerischer, and B. Pettinger. Surface enhanced raman scattering from silver-halide and silver-pyridine vibrations and the role of silver ad-atoms. Chemical Physics Letters, 78(2):392-397, 1981. ISSN 00092614. doi: 10.1016/0009-2614(81)80040-1.

[32] Jing An, Bin Tang, Xianliang Zheng, Ji Zhou, Fengxia Dong, Shuping $\mathrm{Xu}$, Ye Wang, Bing Zhao, and Weiqing $\mathrm{Xu}$. Sculpturing effect of chloride ions in shape transformation from triangular to discal silver nanoplates. Journal of Physical Chemistry C, 112(39):15176-15182, 2008. ISSN 19327447. doi: 10.1021/jp802694p.

[33] Alan Campion and Patanjali Kambhampati. Surface-enhanced Raman scattering. Chemical Society Reviews, 27(4):241-250, 1998. ISSN 
0306-0012. doi: 10.1039/A827241Z. URL http://dx.doi.org/ $10.1039 /$ A $827241 Z$.

[34] Yoshihiro Maruyama and Masayuki Futamata. Anion induced SERS activation and quenching for R6G adsorbed on Ag nanoparticles. Chemical Physics Letters, 448(1-3):93-98, 2007. ISSN 00092614. doi: 10.1016/j.cplett.2007.09.056.

[35] D. Roy and T. E. Furtak. The origin of the potential dependence of the surface enhanced Raman spectrum of chloride on silver. Chemical Physics Letters, 129(5):501-504, 1986. ISSN 00092614. doi: 10.1016/ 0009-2614(86)80236-6.

[36] J. Billmann, G. Kovacs, and A. Otto. Enhanced Raman effect from cyanide adsorbed on a silver electrode. Surface Science, 92(1):153-173, 1980. ISSN 00396028. doi: 10.1016/0039-6028(80)90250-2.

[37] Dean C. Luehrs, Reynold T. Iwamoto, and Jacob Kleinberg. Solubility of Silver Halides and Stability of Silver Halide Complexes in Selected Nonaqueous Media. Inorganic Chemistry, 5(2):201-204, 1966. ISSN 1520510X. doi: 10.1021/ic50036a009. 